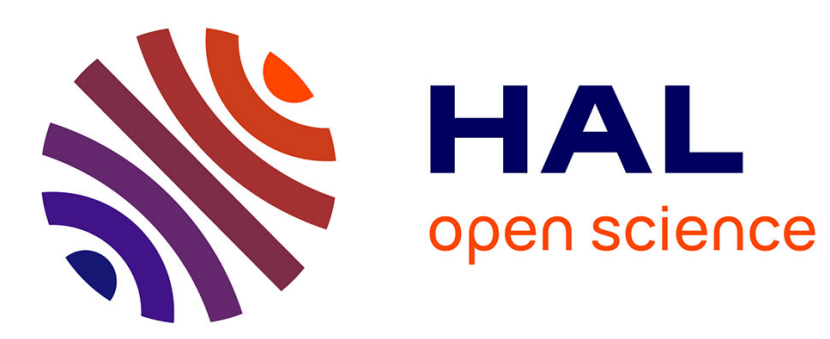

\title{
Exuding porous media: deviations from Darcy's law
}

Didier Lasseux, Francisco Valdés-Parada, Jean-François Thovert, Valeri V.

\author{
Mourzenko
}

\section{To cite this version:}

Didier Lasseux, Francisco Valdés-Parada, Jean-François Thovert, Valeri V. Mourzenko. Exuding porous media: deviations from Darcy's law. Journal of Fluid Mechanics, 2021, 911 (A48), 10.1017/jfm.2020.1081 . hal-03154649

\section{HAL Id: hal-03154649 \\ https://hal.science/hal-03154649}

Submitted on 1 Mar 2021

HAL is a multi-disciplinary open access archive for the deposit and dissemination of scientific research documents, whether they are published or not. The documents may come from teaching and research institutions in France or abroad, or from public or private research centers.
L'archive ouverte pluridisciplinaire HAL, est destinée au dépôt et à la diffusion de documents scientifiques de niveau recherche, publiés ou non, émanant des établissements d'enseignement et de recherche français ou étrangers, des laboratoires publics ou privés. 


\title{
Exuding porous media: Deviations from Darcy's law
}

\author{
Didier Lasseux ${ }^{1} \dagger$, Francisco J. Valdés-Parada ${ }^{2}$, Jean-François \\ Thovert $^{3}$ and Valeri Mourzenko ${ }^{3}$ \\ ${ }^{1}$ I2M, UMR 5295, CNRS, Univ. Bordeaux, Esplanade des Arts et Métiers \\ 33405 Talence CEDEX, France \\ ${ }^{2}$ Universidad Autónoma Metropolitana-Iztapalapa, Departamento de Ingeniería de Procesos e \\ Hidráulica, Av. San Rafael Atlixco 186, 09340 Ciudad de México, Mexico \\ ${ }^{3}$ Institut P', CNRS - Université de Poitiers - ISAE-ENSMA, 11 bd Marie et Pierre Curie, TSA \\ 41123, 86073 POITIERS CEDEX 9, France.
}

(Received xx; revised xx; accepted xx)

This work addresses the question of a pertinent macroscale model describing creeping, incompressible and single-phase flow of a Newtonian fluid in an exuding, rigid and homogeneous porous medium. The macroscopic model is derived by upscaling the porescale Stokes equations considering a normal mass flux at the solid-fluid interface. The upscaled mass equation shows that the average velocity is non-solenoidal. In addition, the macroscopic momentum equation involves a Darcy term with the classical permeability tensor accounting for macroscopic drag and a correction velocity vector which is a signature of the local fluid displacements induced by the exuding phenomenon. This correction is the sum of a term accounting for the local exuding effect and a compensation term associated to the assumption of spatial periodicity. The first term, as well as the permeability tensor, are obtained from the solution of the same unique and intrinsic closure problem, which corresponds to the one involved in classical Darcy's law. The upscaled model is validated by comparisons with pore-scale numerical simulations in several illustrative examples. The different configurations evidence the richness of the problem, despite the apparent simplicity of its formulation. The results of this work motivate further investigation about the influence of internal flow sources in transport phenomena in porous media.

Key words: exuding porous media, momentum transport, upscaling, Darcy's law

\section{Introduction}

Low Reynolds number incompressible flow of a single Newtonian fluid in a rigid porous medium is governed, at the microscopic (pore) scale, by Stokes equations. In the absence of rarefaction effects and of any source or sink, they are supplemented with a zero-velocity condition at the solid-fluid interface. Provided the medium can be regarded as statistically homogeneous at a scale much larger than the typical scale of its microstructure, the flow can be described, from a macroscopic point of view, by Darcy's law. This equation relates the local average of the fluid velocity to the gradient of the average pressure via the intrinsic permeability tensor, which only depends on the geometry of the microstructure

$\dagger$ Email address for correspondence: didier.lasseux@u-bordeaux.fr 
and accounts for the macroscopic drag on the solid phase. Darcy's law can be formally obtained as the macroscopic momentum equation using various theoretical upscaling methodologies, such as homogenization based on multiple scale expansions or the volume averaging theory (see e.g., Sanchez-Palencia 1980; Whitaker 1986; Mei et al. 1996; Davit et al. 2013). As a result of the zero mass-flux condition at the solid-fluid interface at the underlying pore scale, upscaling also yields a divergence-free average velocity as the macroscopic mass conservation equation. Subject to prescribed conditions at the external boundaries of the macroscopic domain, both equations can be used to solve the macroscopic problem.

In a variety of situations, however, the zero mass-flux condition at the solid-fluid interface does not apply. This occurs, for example, when the fluid is released from the solid into the pore space or absorbed from the pore space into the solid, due to many possible mechanisms illustrated in the following. It is then a common practice to account for the volume source in the macroscopic mass conservation equation, while using Darcy's law and the value of the intrinsic permeability, valid in the absence of source (Lachaud et al. 2017; Vu \& Tsotsas 2018). However, the zero velocity at the solid-fluid interface is a key feature in the derivation of the classical form of Darcy's law. There is, a priori, no argument supporting that this equation remains unchanged if non-zero fluxes take place at the interface and, even if a Darcy term remains, the modification or not of the permeability must be carefully addressed. Therefore, it is of interest to determine if such a heuristic formulation is reliable or not. For example, in the presence of interfacial slip, it has been reported that Darcy's law is applicable at the cost that the permeability tensor is no longer intrinsic as it depends on the Knudsen number, in agreement with the fact that the shear is modified by the existence of slip (Lasseux et al. 2016). When a normal flux is present, one may expect that, if a Darcy term is preserved, the permeability remains intrinsic as in classical Darcy's law since the drag is unaltered. However, how the macroscopic momentum equation is affected by the normal inward or outward flow at the pore walls remains to be elucidated. Fundamentally, the problem under consideration raises the questions: $i$ ) Is the solenoidal nature of the macroscopic (average) velocity preserved? ii) Is the Darcy model (macroscopic momentum balance equation) heuristically used for exuding media in some reported works in the literature a physically sound one? iii) If a Darcy term persists in the macroscopic momentum equation, is the permeability still the intrinsic permeability? These questions are addressed in detail in the following sections. This is a very important problem, both from fundamental and potential applications points of view, which, to the best of our knowledge, has not been yet addressed in the literature. Derivation of a valid macroscopic model in such situations is therefore the purpose of the present work.

In the above description, and in some of the following examples, the solid phase is actually often a nanoporous material. Nevertheless, this nanoporous structure is generally too tight to actively participate to the flow within its volume at the scale of description of interest. Consequently, it can be considered as an apparent continuous solid material allowing fluid transfer between its surface and the neighbouring macropores. An example is provided by the exploitation of tight gas reservoirs for which natural or induced fracturing is required to access the resource. The gas contained under high pressure in the nanoporous matrix is exuded at the fracture walls. Its flow towards the producing well through the fractures is described, in field scale simulation models, by Darcy's law (Olorode et al. 2012; Jiang \& Younis 2015), with a permeability which is deduced from the fracture geometrical characteristics in the absence of surface sources (Mourzenko et al. 2018).

Drying or pyrolytic processes also induce interfacial sources, with water vapor or 
pyrolytic gases produced in the solid and exuded into the pore space. Water can be initially chemically bonded or confined in liquid state in nanopores. Pyrolytic gases can result from thermal decomposition of biomass or organic compounds, such as kerogen trapped in the nanopores of oil shales. In the latter case, a largely predominant inert mineral matrix prevents the solid from deforming (Elayeb et al. 2017). Darcy's law and the usual intrinsic permeability are universally used in the macroscopic modelling of such situations, see e.g. (Erriguible et al. 2006; Warning et al. 2015) for drying, (Shepel et al. 2010) for plaster dehydration, (Di Blasi 1994; Larfeldt et al. 2000; Bryden et al. 2002; Mahmoudi et al. 2014) for pyrolysis.

Chemical reactions can also give rise to non-zero velocities at the solid-fluid interface. Consider, for instance, a carbonaceous solid and the heterogeneous reactions $\mathrm{O}_{2}+2 \mathrm{C} \rightarrow$ $2 \mathrm{CO}$ (oxidation) or $\mathrm{CO}_{2}+\mathrm{C} \rightarrow 2 \mathrm{CO}$ (gasification). In both cases, a single mole of $\mathrm{O}_{2}$ or $\mathrm{CO}_{2}$ is consumed and two moles of $\mathrm{CO}$ are produced. This volume increase resulting from the net production of gas localized at the solid surface, can be accounted for by a normal velocity directed into the pores. Reactions between gaseous species can result in the same behaviour, if they require a catalyst contained in the solid, such as, for example, the methanation reaction $\mathrm{CO}_{2}+4 \mathrm{H}_{2} \rightarrow \mathrm{CH}_{4}+2 \mathrm{H}_{2} \mathrm{O}$, or the inverse process of steam reforming (Rönsch et al. 2016). By converting 5 moles of gas into 3 or conversely, it induces a velocity at the surface directed towards or from the solid, respectively.

Fluxes at the pore/solid interface can also result from phase changes. Interesting examples are provided by the water ice/vapour system, with sublimation/condensation at the solid surface. The equilibrium vapour pressure depends on the surface curvature as described by Kelvin's law, giving rise to the isothermal snow ageing metamorphism, with vapour migration from surface sources to surface sinks (Flin et al. 2003; Vetter et al. 2010). It also depends on temperature. Thus, in a bubble enclosed in an ice mass subject to a temperature gradient, a water vapour transfer takes place from a surface source by sublimation on the warm side to a surface sink by condensation on the cold side. This phenomenon, in turn, induces the slow migration of the bubble (Shreve 1967; Dadic et al. 2010).

In many of the above examples, the solid geometry is subject to evolution, by either mass loss or gain inducing shrinkage or growth. However, the characteristic time associated to the deformation is usually very large compared to the one associated to the fluid displacement, so that the flow problem can be treated with a quasi-steady approximation within a fixed geometry. In the water ice/vapour system for instance, this can be explained by the very large solid/gas density contrast.

As a generic problem accounting for the above mentioned situations, the interest is focused in this work on the macroscopic description of flow in a porous material resulting from exuding at the solid-fluid interface at the pore scale, i.e., from a non-zero velocity normal component at this interface. Exuding is meant here in a general sense including fluid release from the surface into the pores or, conversely, absorption from the pore into the solid through the interface. The key question then arises whether Darcy's law is still a valid model at the macroscopic scale, keeping the hypotheses of a Newtonian fluid and creeping incompressible flow conditions.

To address this issue, the article is organized as follows. In section 2, the pore-scale model is presented, the solution of which is illustrated in a simple case, showing that the classical Darcy's law fails and hence, highlighting the necessity of deriving a macroscopic model. Section 3 is dedicated to the upscaling of the pore-scale problem which yields macroscopic mass and momentum transport equations. The former involves a term accounting for the net mass that is released (or absorbed) through the solid-fluid interface and the latter contains a correction term to Darcy's law. This term involves a local 


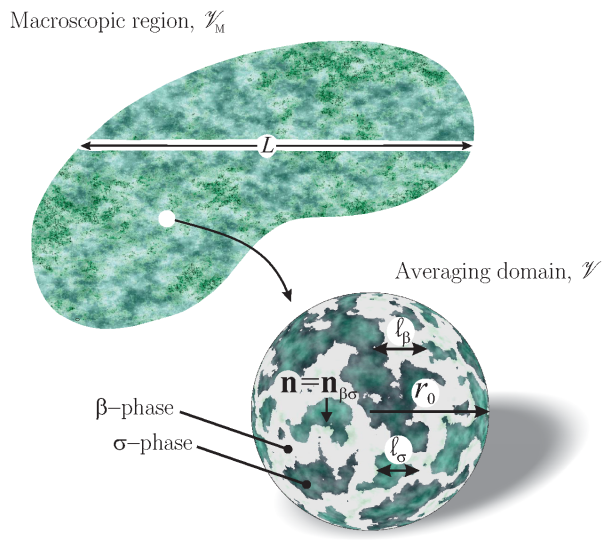

a)

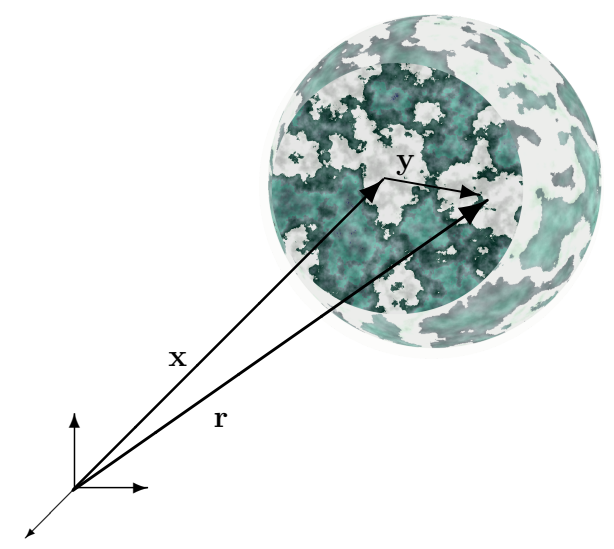

b)

Figure 1. a) Schematic representation of a porous medium saturated with a single fluid phase along with the averaging domain and characteristic lengths. b) Position vectors associated to the averaging volume.

contribution of the exuding effect and a compensation for the periodicity assumption. The model is validated in section 4 by comparisons with pore-scale numerical simulations in a variety of physical situations. Discussion and concluding remarks are provided in section 5 .

\section{Pore-scale model}

In this section, the boundary value problem under consideration and an illustrative analytical solution in a simple case, justifying the analysis proposed in the following sections, are provided.

\subsection{Pore-scale boundary value problem}

Consider a rigid and homogeneous porous medium, $\mathscr{V}_{M}$, of typical size $L$ and of external macroscopic boundary $\partial \mathscr{V}_{M}$, made of a solid rigid skeleton ( $\sigma$-phase) and saturated by a single fluid ( $\beta$-phase) as sketched in figure 1a. The situation of interest is when the $\beta$ phase is either released or absorbed at the solid-fluid interface $\mathscr{A}_{\beta \sigma}$. For this process, the $\beta$-phase is considered as Newtonian and the flow is supposed to remain in the incompressible creeping regime.

Let $\mathscr{V}$, of measure $V$ and size $r_{0}$, in which the region occupied by the $\beta$-phase is denoted by $\mathscr{V}_{\beta}$ (of measure $V_{\beta}$ ), be a subdomain of $\mathscr{V}_{M}\left(\mathscr{V} \subseteq \mathscr{V}_{M}\right)$ referred to as the averaging domain in the following (see figure 1a). At any point located at $\mathbf{r}=\mathbf{x}+\mathbf{y}$ within $\mathscr{V}_{\beta}$, x denoting the position of the centroid of $\mathscr{V}$ (see figure 1b), the fluid pressure may be decomposed under the form $\left.p\right|_{\mathbf{r}}=\left.\langle p\rangle^{\beta}\right|_{\mathbf{r}}+\left.\tilde{p}\right|_{\mathbf{r}}$ (Gray 1975). Application of the gradient operator, leads to

$$
\left.\nabla p\right|_{\mathbf{r}}=\left.\nabla\langle p\rangle^{\beta}\right|_{\mathbf{r}}+\left.\nabla \tilde{p}\right|_{\mathbf{r}}
$$

In these relationships, $\left.\langle\psi\rangle^{\beta}\right|_{\mathbf{r}}$ denotes the intrinsic average of $\psi$ evaluated at $\mathbf{r}$. The averaging operator at any point $\mathbf{x}$ is defined as

$$
\left.\langle\psi\rangle^{\beta}\right|_{\mathbf{x}}=\frac{1}{V_{\beta}} \int_{\mathscr{V}_{\beta}(\mathbf{x})} \psi d V
$$

while $\tilde{\psi}$ represents the deviations of $\psi$ with respect to its intrinsic average. In the course 
of the development, the superficial average defined as

$$
\left.\langle\psi\rangle\right|_{\mathbf{x}}=\frac{1}{V} \int_{\mathscr{V}_{\beta}(\mathbf{x})} \psi d V=\varepsilon\langle\psi\rangle^{\beta}
$$

will also be used, $\varepsilon$ being the porosity of the medium $\left(\varepsilon=V_{\beta} / V\right)$, which is supposed to be constant. This is a reasonable assumption that is supported by the consideration of geometrical homogeneity of the porous medium.

As in the double scale homogenization technique, $\mathbf{x}$ may be understood as the macroscopic (or "slow") variable, having a typical length-scale of variation $L$ while $\mathbf{y}$ corresponds to the microscopic (or "rapid") variable having a characteristic length of variation $\ell_{\beta}$, typical of the pore dimension (see figure $1 \mathrm{~b}$ ). As for any upscaling procedure, a separation of length-scales is assumed, expressed as $\ell_{\beta} \ll r_{0} \ll L$. The gradient of the average pressure evaluated at $\mathbf{r}$ in equation (2.1) may be further considered using the following Taylor series expansion written in a generic form as

$$
\left.\Psi\right|_{\mathbf{r}}=\left.\Psi\right|_{\mathbf{x}}+\left.\mathbf{y} \cdot \nabla \Psi\right|_{\mathbf{x}}+\frac{1}{2} \mathbf{y y}:\left.\nabla \nabla \Psi\right|_{\mathbf{x}}+\ldots
$$

Performing an order of magnitude estimate on the first-order term in this expansion allows writing

$$
\left.\Psi\right|_{\mathbf{r}}=\left.\Psi\right|_{\mathbf{x}}+\mathbf{O}\left(\frac{r_{0}}{L} \Delta \Psi\right)
$$

where $\Delta \Psi$ represents the characteristic variation of $\Psi$ over the length-scale $L$. Since the order of magnitude of $\Delta \Psi$ can be at most as large as $\Psi$, and as a result of the separation of length scales, it seems reasonable to use the approximation $\left.\left.\Psi\right|_{\mathbf{r}} \simeq \Psi\right|_{\mathbf{x}}$. When $\Psi$ represents an average quantity $\langle\psi\rangle^{\beta}$, and, denoting from now on $\left.\langle\psi\rangle^{\beta}\right|_{\mathbf{x}} \equiv\langle\psi\rangle^{\beta}$, this readily implies

$$
\langle\tilde{\psi}\rangle^{\beta} \simeq 0
$$

When $\Psi$ is taken to be $\nabla\langle p\rangle^{\beta}$ and when the resulting approximation is introduced in equation (2.1), the flow problem under consideration can be written in $\mathscr{V}$ as

$$
\begin{gathered}
\nabla \cdot \mathbf{v}=0, \quad \text { in } \mathscr{V}_{\beta} \\
\mathbf{0}=-\frac{1}{\mu} \nabla \tilde{p}+\nabla^{2} \mathbf{v}-\frac{1}{\mu} \nabla\langle p\rangle^{\beta}, \quad \text { in } \mathscr{V}_{\beta} \\
\mathbf{v}=-v_{0} \mathbf{n}, \quad \text { at } \mathscr{A}_{\beta \sigma}
\end{gathered}
$$

In these equations, $\mathbf{v}$ is the velocity in the $\beta$-phase, whose dynamic viscosity, $\mu$, is assumed to be constant. For the sake of simplicity, albeit keeping generality, no body force is considered in the analysis. In the exuding boundary condition at $\mathscr{A}_{\beta \sigma}$, (equation $(2.7 c)), v_{0}$ denotes the rate of production (or consumption) of fluid per unit interfacial area. It is supposed to be a position-dependent but known function of space, a priori, and $\mathbf{n}$ is the unit normal vector at $\mathscr{A}_{\beta \sigma}$ directed from the $\beta$ to the $\sigma$-phase.

Additional boundary conditions at the entrances and exits, $\mathscr{A}_{\beta e}$, of $\mathscr{V}$ must be prescribed in order to complete the problem statement. In a classical homogenization approach, one would certainly consider periodicity at $\mathscr{A}_{\beta}$ as this is the classical way of carrying out the upscaling, assuming locality (Mei et al. 1996). However, taking the superficial average of the mass balance equation (2.7a), applying the divergence theorem 
and making use of the boundary condition at $\mathscr{A}_{\beta \sigma}$, yields

$$
\frac{1}{V} \int_{\mathscr{A}_{\beta e}} \mathbf{n} \cdot \mathbf{v} d A=\varphi_{0}
$$

with

$$
\varphi_{0}=\frac{1}{V} \int_{\mathscr{A}_{\beta \sigma}} v_{0} d A
$$

Clearly, periodicity on $\mathbf{v}$ at $\mathscr{A}_{\beta}$ is not physically admissible unless $\varphi_{0}=0$. Even if it will be further considered later in the development, the boundary condition at $\mathscr{A}_{\beta e}$ is left unspecified at this point. The macroscopic boundary condition over $\mathscr{V}_{M}$ shall nevertheless be denoted as

$$
\mathscr{G}(\mathbf{v}, p)=0, \quad \text { at } \mathscr{A}_{M \beta e}
$$

$\mathscr{A}_{M \beta e}$ denoting the portion of $\partial \mathscr{V}_{M}$ intersecting the fluid phase. Here again, $\mathscr{G}(\mathbf{v}, p)$ must be compliant with the equivalent of equation (2.8) when the entire macroscopic domain is considered.

\subsection{Preliminary comments about the interpretation of Darcy's law}

At this point of the analysis, it is pertinent to make a clear distinction between the superficial average of the pore-scale velocity (i.e., $\langle\mathbf{v}\rangle)$ and another definition of the seepage velocity (denoted $\mathbf{q}_{s}$ in the following) that is typically found in the porous media literature. To this end, let $\langle\mathbf{v}\rangle$ be expressed as follows:

$$
\langle\mathbf{v}\rangle=\frac{1}{V} \int_{\mathscr{V}_{\beta}} \nabla \cdot(\mathbf{v r}) d V-\frac{1}{V} \int_{\mathscr{V}_{\beta}}(\nabla \cdot \mathbf{v}) \mathbf{r} d V
$$

The last term in the above equation is zero due to the solenoidal nature of the pore-scale velocity. Using the divergence theorem in the remaining term, it follows that

$$
\langle\mathbf{v}\rangle=\mathbf{q}_{s}+\frac{1}{V} \int_{\mathscr{A}_{\beta \sigma}} \mathbf{n} \cdot(\mathbf{v r}) d A
$$

with

$$
\mathbf{q}_{s}=\frac{1}{V} \int_{\mathscr{A}_{\beta e}} \mathbf{n} \cdot \mathbf{v r} d A
$$

In the above equations, $\mathbf{q}_{s}$ is recognized in the porous media literature (see, for instance Adler 1992) as the flux or seepage velocity. This definition of the seepage velocity is intuitively appealing and corresponds to a quantity that is easily measurable in the laboratory, with an empirical origin dating back to H. Darcy's pioneering experiments. In the classical Darcy's law, for which the velocity is zero at the solid-fluid interface, the last term in equation (2.12) is zero and it results that $\langle\mathbf{v}\rangle=\mathbf{q}_{s}=-\frac{1}{\mu} \mathbf{K} \cdot \nabla\langle p\rangle^{\beta}$ so that $\langle\mathbf{v}\rangle$ or $\mathbf{q}_{s}$ may be indifferently employed. However, in the case under study, $\mathbf{v}=-v_{0} \mathbf{n}$ at $\mathscr{A}_{\beta \sigma}$ and equation (2.12) takes the form

$$
\langle\mathbf{v}\rangle=\mathbf{q}_{s}-\frac{1}{V} \int_{\mathscr{A}_{\beta \sigma}} v_{0} \mathbf{r} d A
$$

Two important remarks must be made from the expressions of $\langle\mathbf{v}\rangle$ and $\mathbf{q}_{s}$. First, it 


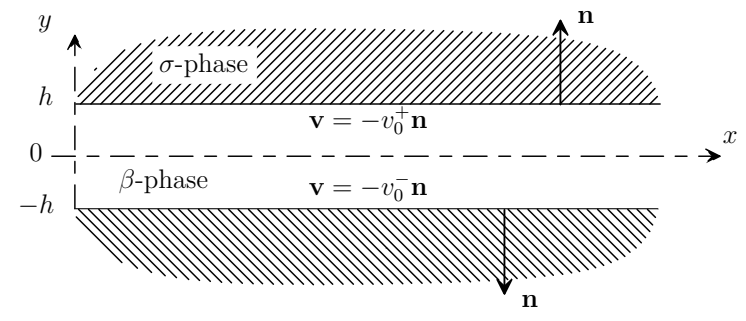

Figure 2. Two-dimensional flat channel made by a pair of plane parallel plates.

should be noted that $\mathbf{q}_{s}$, as given in equation (2.13), is ill-defined because it explicitly depends on the origin of the system of coordinates when there are net sources in the domain. Conversely, the average velocity, $\langle\mathbf{v}\rangle$, does not and, consequently, this is the appropriate definition of the macroscopic velocity that must be used in an upscaled equation. Second, the definition of $\langle\mathbf{v}\rangle$ captures both the mean flow entering/leaving the porous medium $\left(\mathbf{q}_{s}\right)$ and the local fluid displacements resulting from the interfacial sources and/or sinks. These local phenomena must be taken into account for a complete macroscopic description of the flow. The derivations presented in what follows are hence given in terms of $\langle\mathbf{v}\rangle$ and not of $\mathbf{q}_{s}$, which are different in the case under consideration.

\subsection{Solution for a two-dimensional flat channel}

Before developing the macroscopic model and in order to illustrate the specific features of the problem, it is instructive to investigate a simple case for which an analytical solution can be achieved. To this end, a two-dimensional periodic layered medium is considered. Its unit cell contains a channel with aperture $2 h$ as illustrated in figure 2 . The surface sources are denoted $v_{0}^{+}$and $v_{0}^{-}$at $y=h$ and $y=-h$, respectively, and are assumed to be independent of $x$, so that $\varphi_{0}=\frac{\varepsilon}{2 h}\left(v_{0}^{+}+v_{0}^{-}\right)$.

The analytical solution is derived imposing $v_{x}=0$ at $x=0$ with the idea that this is a plane of horizontal symmetry resulting from the fact that the channel is open to the atmosphere at both ends along $x$. The dimensionless solution for the horizontal and vertical velocities, $v_{x}^{*}$ and $v_{y}^{*}$, and pressure, $p^{*}$, in the unit cell is obtained using $2 h, v_{\text {ref }}=\max \left(\left|v_{0}^{+}\right|,\left|v_{0}^{-}\right|\right)$and $\mu \frac{v_{r e f}}{2 h}$ as the reference length, velocity and pressure, respectively. This solution is given by

$$
\begin{gathered}
v_{x}^{*}=\frac{3}{2} \frac{\varphi_{0}^{*}}{\varepsilon}\left(1-4 y^{* 2}\right) x^{*} \\
v_{y}^{*}=-\frac{3}{2} \frac{\varphi_{0}^{*}}{\varepsilon}\left(1-\frac{4}{3} y^{* 2}\right) y^{*}-\frac{v_{0}^{+*}-v_{0}^{-*}}{2} \\
p^{*}=p_{1}^{*}+6 \frac{\varphi_{0}^{*}}{\varepsilon}\left(y^{* 2}-x^{* 2}\right)
\end{gathered}
$$

$p_{1}^{*}$ in equation $(2.15 c)$ being an arbitrary constant. If $\varphi_{0}^{*}$ is positive, the pressure decreases with $x^{*}$ and from the walls to the channel mid-plane. The opposite applies if $\varphi_{0}^{*}$ results in a net sink and $p^{*}$ remains constant if there is no net source or sink.

Although $v_{x}^{*}$ can be identified as a Poiseuille-like solution at each position $x^{*}$, the flow remains two-dimensional. This contrast with respect to the classical flow induced by a pressure gradient along the channel may be better highlighted with the average expressions of $v_{x}^{*}, v_{y}^{*}$ and $p^{*}$ given by

$$
\left\langle v_{x}^{*}\right\rangle^{\beta}=-\frac{1}{12} \frac{\partial\left\langle p^{*}\right\rangle^{\beta}}{\partial x^{*}}
$$




$$
\begin{aligned}
\left\langle v_{y}^{*}\right\rangle^{\beta} & =-\frac{v_{0}^{+*}-v_{0}^{-*}}{2} \\
\left\langle p^{*}\right\rangle^{\beta} & =p_{2}^{*}-6 \frac{\varphi_{0}^{*}}{\varepsilon} x^{* 2}
\end{aligned}
$$

with $\left\langle\psi^{*}\right\rangle^{\beta}=\int_{-1 / 2}^{1 / 2} \psi^{*} d y$ and $p_{2}^{*}=p_{1}^{*}+\varphi_{0}^{*} /(2 \varepsilon)$. Equation $(2.16 a)$, once multiplied by $\varepsilon$, corresponds to the classical Darcy's law, involving the dimensionless permeability, $\varepsilon / 12$, of the channel system in the $x$-direction. For the $y$-component of the velocity, no analogy is possible with a Darcy-like flow. Note that this term accounts for the local fluid displacements and remains even if $\varphi_{0}=0$.

This simple example is a clear evidence that the upscaled model accounting for the presence of surface sources and/or sinks at the solid-fluid interfaces can not be reduced, in general, to Darcy's law.

\section{Upscaling}

In this section, the derivation of a macroscopic model for the flow problem given in equations (2.7) is proposed. The macroscale mass equation is obtained following the classical volume averaging method (Whitaker 1999). However, for the derivation of the upscaled momentum equation, an approach inspired by the adjoint homogenization method recently proposed by Bottaro (2019) is used.

\subsection{Mass balance equation}

In order to derive the macroscopic mass equation, it is convenient to recall the result given in equation (2.8) and use the relationship reported by Slattery (1967),

$$
\frac{1}{V} \int_{\mathscr{A}_{\beta e}} \mathbf{n} \cdot \mathbf{v} d A=\nabla \cdot\langle\mathbf{v}\rangle
$$

in order to obtain

$$
\nabla \cdot\langle\mathbf{v}\rangle=\varphi_{0}
$$

$\varphi_{0}$ being given in equation (2.9). It should be noticed that, except when $\varphi_{0}=0$, this result contrasts with the macroscopic mass balance equation obtained while deriving the classical Darcy's law with a zero velocity at $\mathscr{A}_{\beta \sigma}$ (Whitaker 1986,1999 ) or when a slip flow boundary condition is considered (Lasseux et al. 2016). In other words, a consequence of the conservation of mass within the averaging domain is that $\langle\mathbf{v}\rangle$ has a non-solenoidal character, despite the fact that the flow is incompressible at the underlying pore-scale. This non-solenoidal nature of the average velocity is an important modification to the classical Darcy model as it accounts for the mass entering/exiting the system through the solid-fluid interface. At this point, the macroscopic model must be complemented with the momentum balance equation as proposed in the following paragraphs.

\subsection{Momentum equation}

An efficient and simple way to derive a macroscopic form of the momentum equation, is to make use of the adjoint method proposed by Bottaro (2019). To this purpose, an adjoint problem can be conveniently defined with the minimum requirements as follows

$$
\nabla \cdot \mathbf{D}=\mathbf{0}, \quad \text { in } \mathscr{V}_{\beta}
$$




$$
\begin{gathered}
\mathbf{0}=-\nabla \mathbf{d}+\nabla^{2} \mathbf{D}+\mathbf{I}, \quad \text { in } \mathscr{V}_{\beta} \\
\mathbf{D}=\mathbf{0}, \quad \text { at } \mathscr{A}_{\beta \sigma}
\end{gathered}
$$

Again, boundary conditions for $\mathbf{D}$ and $\mathbf{d}$ at $\mathscr{A}_{\beta e}$ are necessary for this problem to be well-posed. However, they are not specified here as they are not required at this point of the development.

The derivation is carried out by adding the product of equation $(2.7 a)$ by $\mathbf{d}$ to equation $(2.7 b)$ pre-multiplied by $\mathbf{D}^{T}$. Forming the superficial average of the result leads to

$$
\langle\mathbf{d} \nabla \cdot \mathbf{v}\rangle+\left\langle\mathbf{D}^{T} \cdot\left(-\frac{1}{\mu} \nabla \tilde{p}+\nabla^{2} \mathbf{v}\right)\right\rangle=\frac{1}{\mu}\left\langle\mathbf{D}^{T}\right\rangle \cdot \nabla\langle p\rangle^{\beta}
$$

The three terms on the left-hand side of the above equation can be respectively rewritten as

$$
\begin{gathered}
\langle\mathbf{d} \nabla \cdot \mathbf{v}\rangle=\langle\nabla \cdot(\mathbf{v d})\rangle-\langle\mathbf{v} \cdot \nabla \mathbf{d}\rangle \\
-\frac{1}{\mu}\left\langle\mathbf{D}^{T} \cdot \nabla \tilde{p}\right\rangle=-\frac{1}{\mu}\langle\nabla \cdot(\tilde{p} \mathbf{D})\rangle \\
\left\langle\mathbf{D}^{T} \cdot \nabla^{2} \mathbf{v}\right\rangle=\left\langle\mathbf{v} \cdot \nabla^{2} \mathbf{D}\right\rangle+\left\langle\nabla \cdot\left(\nabla \mathbf{v} \cdot \mathbf{D}-\mathbf{v} \cdot(\nabla \mathbf{D})^{T 1}\right)\right\rangle
\end{gathered}
$$

Equation $(3.5 b)$ is obtained while taking into account the divergence-free property of D. Note that in equation $(3.5 c)$, the superscript $T 1$ denotes the transpose operator on the third order tensor $\nabla \mathbf{D}$ which permutes the first and second indices, i.e. $(\nabla \mathbf{D})_{i j k}^{T 1}=$ $(\nabla \mathbf{D})_{j i k}$. Introducing these expressions into equation (3.4) and making use of equation $(3.3 b)$, allows expressing $\langle\mathbf{v}\rangle$ as

$$
\langle\mathbf{v}\rangle=-\frac{1}{\mu}\left\langle\mathbf{D}^{T}\right\rangle \cdot \nabla\langle p\rangle^{\beta}+\left\langle\nabla \cdot\left[\mathbf{v} \cdot\left(\mathbf{I d}-(\nabla \mathbf{D})^{T 1}\right)+\left(-\frac{1}{\mu} \tilde{p} \mathbf{l}+\nabla \mathbf{v}\right) \cdot \mathbf{D}\right]\right\rangle
$$

This last expression can be reformulated using the divergence theorem together with the boundary conditions for $\mathbf{v}$ and $\mathbf{D}$ at $\mathscr{A}_{\beta \sigma}$ given in equations $(2.7 c)$ and $(3.3 c)$ in order to obtain

$$
\begin{aligned}
\langle\mathbf{v}\rangle= & -\frac{1}{\mu}\langle\mathbf{D}\rangle^{T} \cdot \nabla\langle p\rangle^{\beta}-\frac{1}{V} \int_{\mathscr{A}_{\beta \sigma}} v_{0}(\mathbf{d}-\mathbf{n n}: \nabla \mathbf{D}) d A \\
& +\frac{1}{V} \int_{\mathscr{A}_{\beta e}} \mathbf{n} \cdot\left(-\frac{1}{\mu} \tilde{p} \mathbf{l}+\nabla \mathbf{v}\right) \cdot \mathbf{D} d A-\frac{1}{V} \int_{\mathscr{A}_{\beta e}} \mathbf{n} \cdot\left[\mathbf{v} \cdot\left(-\mathbf{l d}+(\nabla \mathbf{D})^{T 1}\right)\right] d A
\end{aligned}
$$

Here, the fact that $\mathbf{n n}: \nabla \mathbf{D}^{T 1}=\mathbf{n n}: \nabla \mathbf{D}$ was taken into account as a result of the symmetry property of the tensor $\mathbf{n n}$. It must be noted that equation (3.7) is valid whatever the boundary conditions at $\mathscr{A}_{\beta}$ are imposed for both the physical (equations (2.7)) and adjoint (equations (3.3)) problems and that the only approximation is the one associated to equation (2.5).

To make further progress towards a closed form of this macroscopic momentum equation, it is now assumed that the porous structure can be assimilated to a periodic one and that $v_{0}$ is also periodic. Under theses circumstances, $\mathscr{V}$ is selected as the smallest periodic unit cell satisfying these properties. Moreover, periodic boundary conditions on $\mathbf{D}$ and $\mathbf{d}$ can be considered for the adjoint problem as this does not imply any physical or mathematical contradiction. This choice is retained in the remainder of the development. Nevertheless, appropriate boundary conditions at $\mathscr{A}_{\beta e}$ for the physical problem are still required, keeping in mind that periodicity on $\mathbf{v}$ is incompatible with equation (2.8) when 
$\varphi_{0} \neq 0$. To handle this situation, it is proposed, as an ansatz, to consider periodic boundary conditions for both $\mathbf{v}$ and $\tilde{p}$ at $\mathscr{A}_{\beta}$ and then, compensate for this assumption as it is explained below. For the moment, it suffices to note that, on the basis of periodic boundary conditions for both the physical and adjoint problems, equation (3.7) reduces to

$$
\langle\mathbf{v}\rangle=-\frac{1}{\mu}\left\langle\mathbf{D}^{T}\right\rangle \cdot \nabla\langle p\rangle^{\beta}-\frac{1}{V} \int_{\mathscr{A}_{\beta \sigma}} v_{0} \mathbf{d} d A+\frac{1}{V} \int_{\mathscr{A}_{\beta \sigma}} v_{0} \mathbf{n n}: \nabla \mathbf{D} d A
$$

An additional simplification can be made regarding the last term in the above expression of $\langle\mathbf{v}\rangle$. Taking into account the fact that $\mathbf{v}$ is a solenoidal field allows writing

$$
\left\langle\mathbf{D}^{T} \cdot\left(\nabla \cdot(\nabla \mathbf{v})^{T}\right)\right\rangle=\left\langle\nabla \cdot\left((\nabla \mathbf{v})^{T} \cdot \mathbf{D}-\mathbf{v} \cdot \nabla \mathbf{D}\right)\right\rangle=\mathbf{0}
$$

Applying the divergence theorem to the term in the middle of this equation (3.9) leads to

$$
\frac{1}{V} \int_{\mathscr{A}_{\beta \sigma}} v_{0} \mathbf{n n}: \nabla \mathbf{D} d A+\frac{1}{V} \int_{\mathscr{A}_{\beta e}} \mathbf{n} \cdot(\nabla \mathbf{v})^{T} \cdot \mathbf{D} d A-\frac{1}{V} \int_{\mathscr{A}_{\beta e}} \mathbf{n} \cdot(\mathbf{v} \cdot \nabla \mathbf{D}) d A=\mathbf{0}
$$

When periodic boundary conditions for both $\mathbf{v}$ and $\mathbf{D}$ are considered, the two last terms on the left hand side of this last relationship are zero, which means that

$$
\frac{1}{V} \int_{\mathscr{A}_{\beta \sigma}} v_{0} \mathbf{n n}: \nabla \mathbf{D} d A=\mathbf{0}
$$

This allows simplifying equation (3.8) to

$$
\langle\mathbf{v}\rangle=-\frac{1}{\mu}\langle\mathbf{D}\rangle^{T} \cdot \nabla\langle p\rangle^{\beta}-\frac{1}{V} \int_{\mathscr{A}_{\beta \sigma}} v_{0} \mathbf{d} d A
$$

It must be kept in mind that this form is an approximation of $\langle\mathbf{v}\rangle$, as a result of the application of periodic boundary conditions for $\mathbf{v}$ at $\mathscr{A}_{\beta e}$. Moreover, at this stage of the derivations, an important point remains to be carefully addressed. Indeed, the adjoint problem for $\mathbf{D}$ and $\mathbf{d}$ given in equations (3.3), complemented with periodic boundary conditions, remains ill-posed as $\mathbf{d}$ can only be determined to within an arbitrary additive constant, and this leaves the expression of $\langle\mathbf{v}\rangle$ undetermined due to the last integral term on the right hand side of equation (3.12). When $v_{0}$ is uniformly zero, i.e., when the problem reduces to the derivation of Darcy's law, it can be shown that an additional constraint can be imposed on $\mathbf{d}$ by setting $\langle\mathbf{d}\rangle^{\beta}$ equal to an arbitrary constant for the adjoint problem to be well-posed. This is due to the fact that, in this particular case, the closed macroscopic momentum equation only involves $\langle\mathbf{D}\rangle$ and that the field of $\mathbf{D}$ is insensitive to any additive constant superimposed to the field of $\mathbf{d}$. Indeed, the only source of the physical problem originates from the gradient of the average pressure reflected in the source term $\mathbf{I}$ in equation $(3.3 b)$. However, when a non-zero exuding flux is present, $\mathbf{d}$ must be uniquely defined in equation (3.12) to obtain a closed expression for $\langle\mathbf{v}\rangle$. This feature results from the fact that periodic boundary conditions have been considered for $\mathbf{v}$, while the solution for $\langle\mathbf{v}\rangle$ depends on both the internal surface source or sink, $v_{0}$, and the macroscopic boundary conditions. To circumvent this difficulty, it is convenient to consider the decomposition of $\mathbf{d}$ under its intrinsic average $\boldsymbol{\lambda}=\langle\mathbf{d}\rangle^{\beta}$ and deviation $\mathbf{d}_{1}=\tilde{\mathbf{d}}$ as

$$
\mathbf{d}=\boldsymbol{\lambda}+\mathbf{d}_{1}
$$


Keeping in mind that, within the periodic unit cell, $\mathscr{V},\left\langle\mathbf{d}_{1}\right\rangle^{\beta}=\mathbf{0}$ (see equation (2.6) with $\tilde{\psi}=\mathbf{d}_{1}$ ) and that $\boldsymbol{\lambda}$ can be considered as a constant (see equation (2.5) with $\Psi=\boldsymbol{\lambda}$ together with the ensuing approximation), the adjoint problem for $\mathbf{d}_{1}$ and $\mathbf{D}$ can be written as

$$
\begin{gathered}
\nabla \cdot \mathbf{D}=\mathbf{0}, \quad \text { in } \mathscr{V}_{\beta} \\
\mathbf{0}=-\nabla \mathbf{d}_{1}+\nabla^{2} \mathbf{D}+\mathbf{I}, \quad \text { in } \mathscr{V}_{\beta} \\
\mathbf{D}=\mathbf{0} \quad \text { at } \mathscr{A}_{\beta \sigma} \\
\psi\left(\mathbf{r}+\mathbf{l}_{i}\right)=\psi(\mathbf{r}), \quad i=1,2,3 ; \psi=\mathbf{d}_{1}, \mathbf{D} \\
\left\langle\mathbf{d}_{1}\right\rangle^{\beta}=\mathbf{0}
\end{gathered}
$$

where $\mathbf{l}_{i}$ are the periodic lattice vectors of $\mathscr{V}$. This problem exactly corresponds to the closure problem ( $\mathbf{D}$ and $\mathbf{d}_{1}$ being the closure variables) involved in the derivation of the classical Darcy's law from which the intrinsic permeability tensor $\mathbf{K}$ is given by (Whitaker 1986, 1999; Mei et al. 1996; Mei \& Vernescu 2010)

$$
\mathbf{K}=\langle\mathbf{D}\rangle
$$

Introduction of the decomposition for $\mathbf{d}$ into equation (3.12) while taking into account that $\mathbf{K}$ is a symmetric tensor (see e.g. Lasseux \& Valdés-Parada 2017), yields

$$
\langle\mathbf{v}\rangle=-\frac{1}{\mu} \mathbf{K} \cdot \nabla\langle p\rangle^{\beta}+\mathbf{v}_{s}
$$

where the vector $\mathbf{v}_{s}$, denoting the correction velocity to Darcy's law due to the surface source or sink, $v_{0}$, is defined as

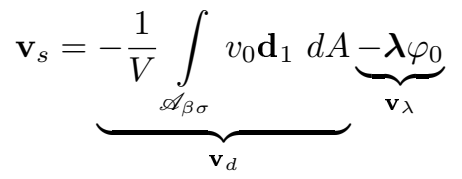

It should be noted that both $\mathbf{K}$ and $\mathbf{v}_{d}$ are obtained from the same closure (adjoint) problem that is intrinsic to the structure under consideration and it corresponds to the same closure problem yielding $\mathbf{K}$ in the classical Darcy's law. This is novel in the volume averaging method, where it is usually required to produce a distinct closure problem for each source in the pore-scale model. In addition, it should be noted that $\mathbf{v}_{d}$, like $\mathbf{K}$, is independent of the fluid viscosity, but, unlike $\mathbf{K}$, it is unmodified in a homothetic change of scale of the microstructure.

To clarify the physical meaning of the terms involved in the macroscopic momentum equation (3.16), it is instructive to rewrite it in the following alternative form

$$
-\frac{\mathbf{K}}{\mu} \cdot \nabla\langle p\rangle^{\beta}=-\frac{\mathbf{K}}{\mu} \cdot \frac{1}{V_{\beta}} \int_{\mathscr{A}_{\beta \sigma}} \mathbf{n} \cdot \mathbf{T} d A=\mathbf{v}_{1}=\langle\mathbf{v}\rangle-\mathbf{v}_{s}
$$

where $\mathbf{T}$ is the total stress tensor $\left(\mathbf{T}=-p \mathbf{I}+\mu\left(\nabla \mathbf{v}+\nabla \mathbf{v}^{T}\right)\right)$. In this formulation, $\mathbf{v}_{1}$, which identifies as the Darcy term, corresponds to the average contribution of the microscale flow field that induces a force on the solid phase (which translates into the macroscopic pressure gradient). Conversely, $\mathbf{v}_{s}$ is the contribution that does not induce such a force but is nevertheless not zero due to the local fluid displacements.

In the expression of $\mathbf{v}_{s}$, the first term (i.e., $\mathbf{v}_{d}$ ) accounts for the local effect of $v_{0}$ whereas the second term $\left(i . e ., \mathbf{v}_{\lambda}\right.$ ) is a compensation of the effect of the periodic boundary condition applied to $\mathbf{v}$ at the level of the unit cell. The idea here is that $\boldsymbol{\lambda}$ can be computed 
from equation (3.16) with $\langle\mathbf{v}\rangle$ obtained from solving the pore-scale flow problem in a domain potentially significantly smaller than $\mathscr{V}_{M}$ with the same macroscopic boundary condition (see equation (2.10)). If such reduction is not possible, then it should be evaluated from a flow solution over the entire domain. Nevertheless, as illustrated in section 4 below, $\boldsymbol{\lambda}$ has no significant contribution in many circumstances, even if it can be crucial in some very specific cases. The correction term $\mathbf{v}_{\lambda}$ in $\mathbf{v}_{s}$ does not even exist when $\varphi_{0}=0$ and this is consistent with the fact that, in that case, periodic boundary conditions for $\mathbf{v}$ at $\mathscr{A}_{\beta e}$ are physically admissible.

A particular feature of $\mathbf{v}_{d}$ must be pointed out when the structure is non-percolating in a direction, $\mathbf{e}_{k}$, of space. In such circumstances, the projection of the closure problem on $\mathbf{e}_{k}$ admits the unique solution

$$
\mathbf{D} \cdot \mathbf{e}_{k}=\mathbf{0}
$$

and consequently

$$
\mathbf{d}_{1} \cdot \mathbf{e}_{\mathbf{k}}=\left(\mathbf{r}-\mathbf{r}_{G}\right) \cdot \mathbf{e}_{k}
$$

$\mathbf{r}_{G}$ being the barycentre of $\mathscr{V}_{\beta}$. From equation $(3.19 b)$, it appears that $\mathbf{v}_{d} \cdot \mathbf{e}_{k}$ is the first moment of $v_{0}$ in the $\mathbf{e}_{k}$ direction. Hence, an average velocity may be induced in a nonpercolating direction of the medium in the absence of a macroscopic pressure gradient, as already noted in the case of the flat channel (see equation $(2.16 b)$ ). This is further illustrated in section 4 .

The fact that the correction to Darcy's law given in equation (3.16) is a vector implies that, for situations in which the microstructure and the distribution of $v_{0}$ are both isotropic, $\mathbf{v}_{s}$ should be $\mathbf{0}$ due to the absence of a privileged direction. Isotropy can also result from statistical reasons, such as the averaging of the randomness in real media. In addition, $\mathbf{v}_{d}$ may also be evanescent because of an uncorrelated combination of $\mathbf{d}_{1}$ and $v_{0}$. In other words, $\mathbf{v}_{s}$ plays a role only if there is some spatial alignment in the local geometrical and/or fluid exuding distribution properties, in particular, when $v_{0}$ presents some kind of polarization between releasing and absorbing interfaces. Moreover, an order of magnitude estimate can be performed on $\mathbf{v}_{d}$ and $\mathbf{v}_{\lambda}$ as reported in Appendix A. It shows that both $\mathbf{v}_{d}$ and $\mathbf{v}_{\lambda}$ are, at most, of order $v_{0}$.

As a summary, the upscaled (or macroscopic) model describing flow in porous media of a Newtonian fluid in the creeping incompressible regime, induced by a rigid and homogeneous exuding solid matrix, is given by

$$
\begin{gathered}
\nabla \cdot\langle\mathbf{v}\rangle=\varphi_{0} \\
\langle\mathbf{v}\rangle=-\frac{\mathbf{K}}{\mu} \cdot \nabla\langle p\rangle^{\beta}+\mathbf{v}_{s}
\end{gathered}
$$

in which $\varphi_{0}$ and $\mathbf{v}_{s}$ are given by equations (2.9) and (3.17), respectively, and this represents the salient result of this work. Clearly, the macroscopic momentum equation $(3.20 b)$ differs from Darcy's law by the correction term $\mathbf{v}_{s}$, while $\langle\mathbf{v}\rangle$ is not a divergencefree field, except when $\varphi_{0}=0$.

\section{Results}

The purpose of this section is to assess the validity of the macroscale model derived in section 3 for a variety of situations. Firstly, flow in two geometries of corrugated two-dimensional channels is considered for three configurations of $v_{0}$ at the solid-fluid interface. Secondly, flow in both isotropic and anisotropic two-dimensional model porous media is analysed. Finally, the case of exuding within spherical bubbles is investigated, a 
situation which may be thought of as a simple representation of the migration of water vapor bubbles in ice.

In all the situations studied here, the reference macroscale velocity and pressure results are those obtained from averaging the numerical solution of the governing flow equations at the pore-scale carried out in $\mathscr{V}_{M}$. The pore-scale numerical simulation (PSNS) of the flow problem, as well as the solutions of the closure problem given in equation (3.14) and of the upscaled model are carried out using the finite element solver Comsol Multiphysics 5.5. The smoothed aggregation algebraic multigrid solver was chosen for the fluid flow variables considering preconditioning. In addition, typical tests of convergence in terms of the relative tolerance and number of mesh elements were performed so that the results presented here are independent of these numerical degrees of freedom.

For the developments reported below, it is convenient to introduce the following dimensionless variables and parameters

$$
\begin{aligned}
& \mathbf{r}^{*}=\frac{\mathbf{r}}{\ell_{\text {cell }}} ; \quad \mathbf{v}^{*}=\frac{\mathbf{v}}{v_{\text {ref }}} ; \quad v_{0}^{*}=\frac{v_{0}}{v_{\text {ref }}} \quad p^{*}=\frac{p \ell_{\text {cell }}}{\mu v_{\text {ref }}} ; \quad \varphi_{0}^{*}=\frac{\varphi_{0} \ell_{\text {cell }}}{v_{\text {ref }}} ; \quad \mathbf{K}^{*}=\frac{\mathbf{K}}{\ell_{\text {cell }}^{2}} ; \\
& \quad \boldsymbol{\lambda}^{*}=\frac{\lambda}{\ell_{\text {cell }}} ; \quad \mathbf{d}_{1}^{*}=\frac{\mathbf{d}_{1}}{\ell_{\text {cell }}} ; \quad \mathbf{D}^{*}=\frac{\mathbf{D}}{\ell_{\text {cell }}^{2}}
\end{aligned}
$$

Here $v_{\text {ref }}=\max \left(\left|v_{0}\right|\right)$ and $\ell_{\text {cell }}$ is the length of the geometrical periodic unit cell used to construct the solution domain.

Before presenting the numerical results, the analytical solution obtained from the macroscopic model in the case of the flat channel is compared to the flow solution reported in section 2.3 , keeping the same conditions. In that case, $\ell_{\text {cell }}=2 h$ and the analytical solution of equations (3.14) for $\mathbf{D}^{*}$ and $\mathbf{d}_{1}^{*}$ is given by

$$
\begin{gathered}
D_{x x}^{*}=\frac{1}{2}\left(-y^{* 2}+\frac{1}{2}\right), \quad D_{y x}^{*}=0, \quad d_{1 x}^{*}=0 \\
D_{x y}^{*}=0, \quad D_{y y}^{*}=0, \quad d_{1 y}^{*}=y^{*}
\end{gathered}
$$

Note that the results in equations $(4.2 b)$ correspond to the solution in equations (3.19), as expected. Once these results are inserted into equation (3.16), it yields

$$
\begin{gathered}
\left\langle v_{x}^{*}\right\rangle=-\frac{\varepsilon}{12} \frac{\partial\left\langle p^{*}\right\rangle^{\beta}}{\partial x^{*}} \\
\left\langle v_{y}^{*}\right\rangle=-\frac{\varepsilon\left(v_{0}^{+*}-v_{0}^{-*}\right)}{2}
\end{gathered}
$$

Combining the macroscopic mass and momentum equations (3.20), leads to the following average dimensionless pressure equation

$$
-\frac{\varepsilon}{12} \frac{\partial^{2}\left\langle p^{*}\right\rangle^{\beta}}{\partial x^{* 2}}=\varphi_{0}^{*}=\varepsilon\left(v_{0}^{+*}+v_{0}^{-*}\right)
$$

The solution of this equation is obtained by considering that $\left\langle v_{x}^{*}\right\rangle=0$ (i.e. $\partial\left\langle p^{*}\right\rangle^{\beta} / \partial x^{*}=$ $0)$ at $x^{*}=0$ and it can be written as

$$
\left\langle p^{*}\right\rangle^{\beta}=p_{2}^{*}-6 \frac{\varphi_{0}^{*}}{\varepsilon} x^{* 2}
$$

with $p_{2}^{*}$ being an arbitrary constant. The results given in equations (4.3) and $(4.4 b)$ coincide with those obtained from the flow solution (see equations (2.16)). In particular, $\mathbf{v}_{\lambda}^{*}$ is zero and the result from the macroscopic model is exact in that case. 

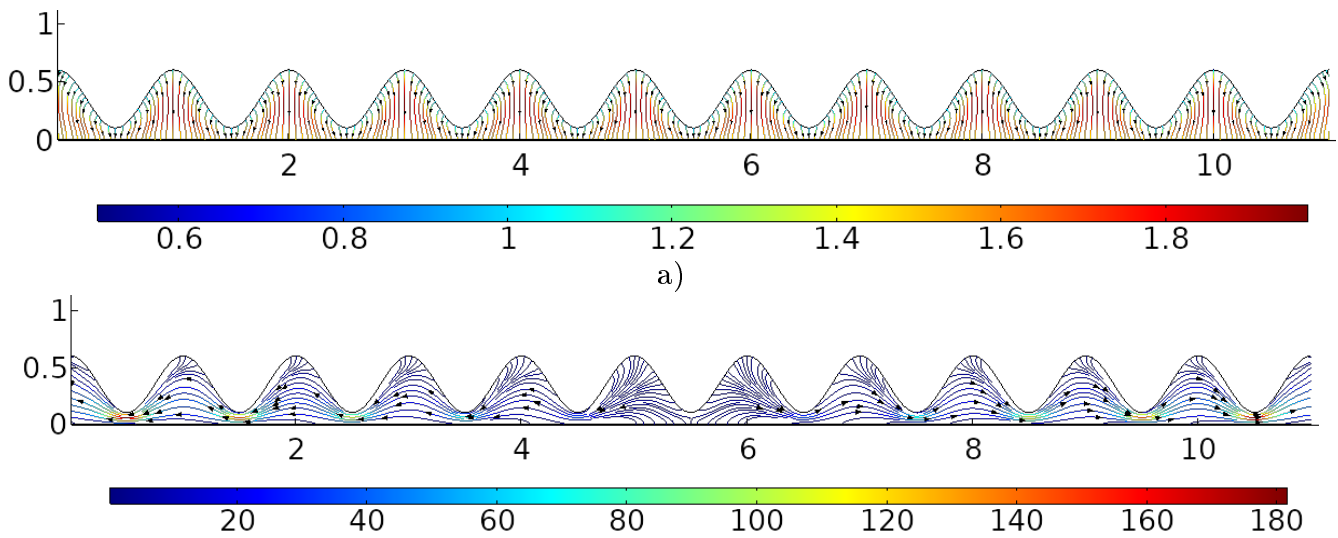

b)

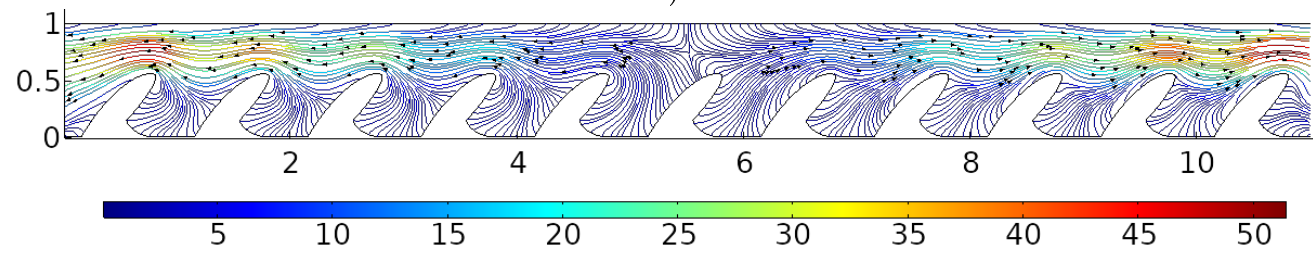

c)

Figure 3. Pore-scale streamlines corresponding to a) Case a, b) Case b and c) Case d. Case c is not reported for the sake of brevity.

\subsection{Flow in corrugated channels}

In this section, two types of two-dimensional channels, for which the macroscopic domain, $\mathscr{V}_{M}$, is composed of the $N$-repetition $(N=11$ here) along $x$ of a geometrical periodic unit cell, are considered. This geometry may be viewed as a simple model to study flow in exuding fractures (cf., Rasoulzadeh et al. 2020). For the first channel geometry, namely the wavy channel, the lower wall at $y^{*}=0$ is flat, while the upper wall follows the oscillatory function $y^{*}=0.35+0.25 \cos \left(2 \pi x^{*}\right)$. The developed dimensionless length of the upper wall is denoted by $A_{\text {upper }}^{*}$. The second type of geometry, referred to as the shark-fin channel, is such that the lower wall is corrugated and not symmetric. It presents a positive undulation over the baseline $y^{*}=0$ while the upper wall is flat, positioned at $y^{*}=1$. In the following numerical evaluations, the dimensionless height of the unit cell is taken equal to the maximum dimensionless channel aperture, i.e., 0.6 and 1 for the wavy and shark-fin channels respectively. The interest is to examine the following four configurations:

Case a $v_{0}^{*}=1$ at the upper wall and $v_{0}^{*}=-A_{u p p e r}^{*}$ at the lower wall in the wavy channel, so that $\varphi_{0}^{*}=0$;

Case $\mathbf{b} v_{0}^{*}=1$ at $\mathscr{A}_{\beta \sigma}$ in the wavy channel;

Case c A Janus-type $\nmid$ configuration for which $v_{0}^{*}=1$ at the solid-fluid interfaces located in the right half and $v_{0}^{*}=0$ in the left half of each geometrical unit cell in the wavy channel;

Case $\mathbf{d} v_{0}^{*}=1$ at $\mathscr{A}_{\beta \sigma}$ in the shark-fin channel.

To complete the problem statement, the following form of $\mathscr{G}^{*}\left(\mathbf{v}^{*}, p^{*}\right)$ for the condition at the macroscopic boundaries, $\mathscr{A}_{M \beta e}$, (see equation $(2.10)$ ) is imposed for all the pore-

$\dagger$ named after the ancient Roman two-faced sculpture. 
TABLE 1. Values of the permeability $\left(\mathbf{K}^{*}\right)$ and the components of $\mathbf{v}_{s}^{*}$ for all the numerical simulation cases. Note that $v_{\lambda x}^{*}$ was found to be irrelevant in all cases.

\begin{tabular}{|c|c|c|c|c|c|c|}
\hline Case & & $\mathbf{K}^{*}$ & $\varphi_{0}^{*}$ & $v_{d x}^{*}$ & $v_{d y}^{*}$ & $v_{\lambda y}^{*}$ \\
\hline $\begin{array}{l}\text { Wavy channel Case a } \\
\text { Wavy channel Case b } \\
\text { Wavy channel Case c }\end{array}$ & $\left(\begin{array}{c}6.14 \\
0\end{array}\right.$ & $\left.\begin{array}{l}0 \\
0\end{array}\right) \times 10^{-4}$ & $\begin{array}{c}0 \\
4.11 \\
2.05\end{array}$ & $\begin{array}{c}0 \\
0 \\
0.401\end{array}$ & $\begin{array}{l}0.854 \\
0.048 \\
0.024\end{array}$ & $\begin{array}{c}0 \\
-0.349 \\
-0.174\end{array}$ \\
\hline Shark-fin channel Case d & $\left(\begin{array}{c}1.18 \\
0\end{array}\right.$ & $\left.\begin{array}{l}0 \\
0\end{array}\right) \times 10^{-2}$ & 3.04 & 0.143 & 0.211 & 0.514 \\
\hline $\begin{array}{l}\text { Isotropic porous medium Case a } \\
\text { Isotropic porous medium Case b } \\
\text { Anisotropic porous medium } \\
\text { vertical Janus } \\
\text { Anisotropic porous medium } \\
\text { horizontal Janus }\end{array}$ & $\begin{array}{l}\left(\begin{array}{c}1.94 \\
0\end{array}\right. \\
\left(\begin{array}{c}31.10 \\
3.31\end{array}\right.\end{array}$ & 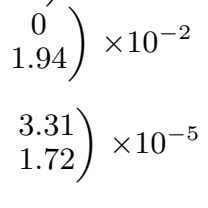 & $\begin{array}{c}0 \\
0.793 \\
4.92 \\
4.81\end{array}$ & $\begin{array}{c}0 \\
0.283 \\
0.039\end{array}$ & $\begin{array}{c}0.404 \\
0 \\
0.346\end{array}$ & $\begin{array}{l}0 \\
0 \\
0\end{array}$ \\
\hline
\end{tabular}

scale numerical simulations

$$
\mathscr{G}^{*}\left(\mathbf{v}^{*}, p^{*}\right)=\mathbf{n} \cdot\left[-p^{*} \mathbf{I}+\nabla^{*} \mathbf{v}^{*}+\left(\nabla^{*} \mathbf{v}^{*}\right)^{T}\right]=\mathbf{0}
$$

with $\mathbf{n}$ being the unit normal vector at $\mathscr{A}_{M \beta e}$ pointing out of the $\beta$-phase. The PSNS were performed considering a channel composed of eleven geometrical unit cells and it was verified that similar results were obtained considering larger values of $N$. In all the simulations, the coordinate axes origin is located at the lower left corner of the system. The pore-scale streamlines for the three cases listed above are presented in figure 3 . Regarding these results the following comments are in order:

(i) In Case a, the streamlines appear to be periodic from one geometric unit cell to another. Since $\varphi_{0}^{*}=0$, fluid is released from the upper wall and absorbed at the lower wall at the same rate so that the net flow is directed from the upper to the lower wall and no overall flow is directed towards $\mathscr{A}_{M \beta e}$. As a matter of fact, similar results are obtained if, instead of equation (4.5), one imposes periodic boundary conditions at $\mathscr{A}_{M \beta e}$, which complies with mass conservation in this case.

(ii) In Cases b and d, there is a net fluid source, with $\varphi_{0}^{*}>0$. Consequently, the fluid tends to move from the channel centre towards $\mathscr{A}_{M \beta e}$. Notice that in Case d, the streamlines do not follow a symmetric pattern: the velocity is larger near the left macroscopic boundary than near the right one.

(iii) The results for Case $\mathrm{c}$ are equivalent to those shown in figure $3 \mathrm{~b}$, except that the maximum values are divided by a factor of two and they are not reported in this figure for the sake of brevity.

The predictions from the macroscopic model derived here are compared with these PSNS for the above four cases. Comparisons are made in terms of the horizontal and vertical components of the velocity vector, $\left\langle\mathbf{v}^{*}\right\rangle$, and the intrinsic average pressure, $\left\langle p^{*}\right\rangle^{\beta}$. It should be noted that a straightforward consequence of the mass equation for these geometries is that the profiles of $\left\langle v_{x}^{*}\right\rangle$ along $x^{*}$ are linear with a slope equal to $\varphi_{0}^{*}$, a feature that was recovered in all the four cases with the PSNS and the macroscale predictions.

A salient feature of the upscaling approach presented in section 3 is that it is necessary to solve only one closure problem (see equations (3.14)) in order to compute the field of $\mathbf{D}^{*}$, from which $\mathbf{K}^{*}$ is obtained and the field of $\mathbf{d}_{1}^{*}$, from which $\mathbf{v}_{d}^{*}$ can be deduced for 


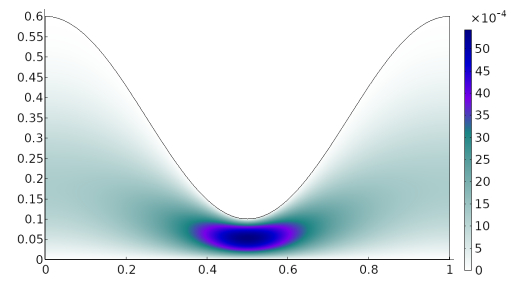

a)

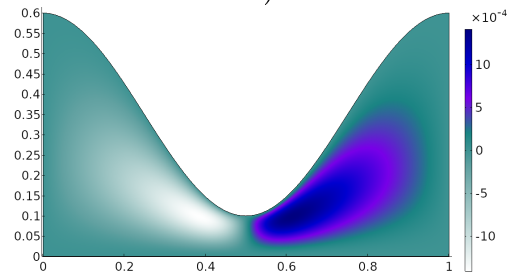

b)

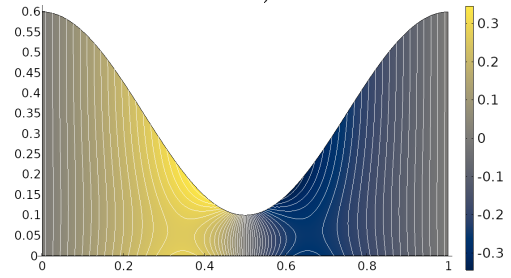

c)

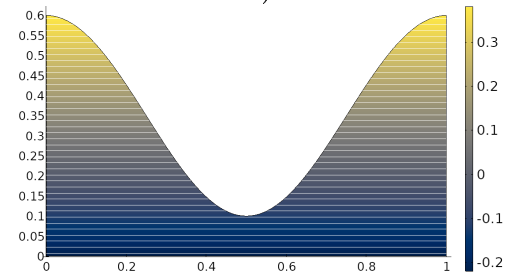

d)

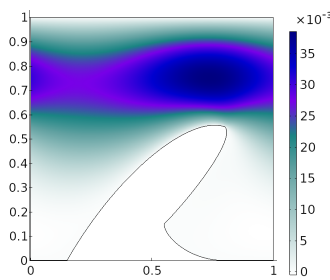

i)

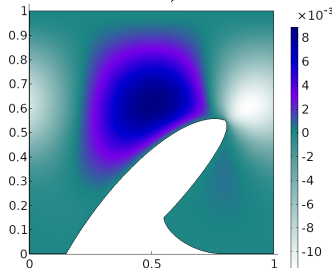

ii)

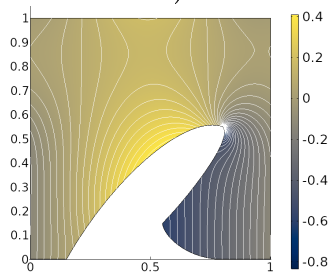

iii)

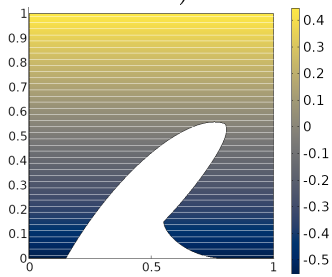

iv)

Figure 4. Fields of the closure variables corresponding to the wavy channel (a-d) and to the shark-fin channel (i-iv). a) and i) $D_{x x}^{*}$; b) and ii) $D_{y x}^{*}$; c) and iii) $d_{1 x}^{*}$ with isolines; d) and iv) $d_{1 y}^{*}$ with isolines. The fields of $D_{x y}^{*}$ and $D_{y y}^{*}$ are zero and are not represented here.

any source distribution. The fields of the closure variables are presented for both channel geometries in figure 4 . In specific, the fields of the $x x$ and $y x$ components of the closure tensor $\mathbf{D}^{*}$ are presented in figures $4 \mathrm{a}, 4 \mathrm{i}$ and $4 \mathrm{~b}, 4 \mathrm{ii}$. Since the channels do not percolate in the $y$-direction, the $x y$ and $y y$ components of $\mathbf{D}^{*}$ are zero for both geometries (see equations $3.19 a$ ). In addition, the fields of the $x$ and $y$ components of the closure variable vector $\mathbf{d}_{1}^{*}$ are shown in figures $4 \mathrm{c}, 4$ iii and $4 \mathrm{~d}$, $4 \mathrm{iv}$. As expected, the fields of $d_{1 y}^{*}$ in figures $4 \mathrm{~d}$ and $4 \mathrm{iv}$ are in agreement with equation $(3.19 b)$. From the solution on $\mathbf{D}^{*}$, it follows that the components of the permeability tensor are $K_{y x}^{*}=K_{x y}^{*}=K_{y y}^{*}=0$ for both channels, while the only non-zero term is $K_{x x}^{*}$. Its values are reported in Table 1.

Under these circumstances, the equation resulting from the combination of the macroscale mass and momentum equations reduces to

$$
K_{x x}^{*} \frac{d^{2}\left\langle p^{*}\right\rangle^{\beta}}{d x^{* 2}}=-\varphi_{0}^{*}
$$


which is subject to the following boundary conditions

$$
\begin{aligned}
\text { at } x^{*}=x_{0}^{*}, & \left\langle p^{*}\right\rangle^{\beta}=p_{0}^{*} \\
\text { at } x^{*}=x_{L}^{*}, & \left\langle p^{*}\right\rangle^{\beta}=p_{L}^{*}
\end{aligned}
$$

Here, $p_{0}^{*}$ and $p_{L}^{*}$ are the values of the dimensionless intrinsic average pressure obtained from the PSNS at the first and last unit cell, located at $x_{0}^{*}=0.5$ and $x_{L}^{*}=10.5$, respectively. The analytical solution of this boundary-value problem is

$$
\left\langle p^{*}\right\rangle^{\beta}=p_{0}^{*}+\frac{\left(p_{L}^{*}-p_{0}^{*}\right)\left(x^{*}-x_{0}^{*}\right)}{\left(x_{L}^{*}-x_{0}^{*}\right)}-\frac{\varphi_{0}^{*}}{2 K_{x x}^{*}}\left[x^{* 2}-x_{0}^{* 2}-\left(x_{L}^{*}+x_{0}^{*}\right)\left(x^{*}-x_{0}^{*}\right)\right]
$$

From the above expression, it is easy to obtain

$$
\frac{d\left\langle p^{*}\right\rangle^{\beta}}{d x^{*}}=\frac{\left(p_{L}^{*}-p_{0}^{*}\right)}{\left(x_{L}^{*}-x_{0}^{*}\right)}-\frac{\varphi_{0}^{*}}{2 K_{x x}^{*}}\left[2 x^{*}-\left(x_{L}^{*}+x_{0}^{*}\right)\right]
$$

which is further used to compute the Darcy term.

The values of the two terms $\mathbf{v}_{d}^{*}$ and $\mathbf{v}_{\lambda}^{*}$ change for each case study as reported in Table 1. Nevertheless, some insight can be obtained from the results in figure $4 \mathrm{c}$. In this plot, it is observed that $d_{1 x}^{*}$ is antisymmetric around $x^{*}=0.5$ at both solid-fluid interfaces. This means that the contributions from $v_{d x}^{*}$ should be zero for the wavy channel when $v_{0}^{*}$ is uniform at $\mathscr{A}_{\beta \sigma}$. However, this is not true for the shark-fin channel. In this configuration, the contributions from both $d_{1 x}^{*}$ and $d_{1 y}^{*}$ are relevant because no particular antisymmetry condition is observed. Furthermore, since the permeability is zero in the $y$-direction, it follows that $v_{s y}^{*}$ should be the only relevant term in the predictions of $\left\langle v_{y}^{*}\right\rangle$ in both channel geometries.

Regarding the predictions of $\boldsymbol{\lambda}^{*}$, its values are obtained from a combination of the macroscale model and PSNS in a reduced macroscale domain. As mentioned in section 3.2 , the pore-scale equations can be solved in a subdomain of $\mathscr{V}_{M}$ containing either three or five geometric unit cells in order to obtain the values of $\left\langle v_{x}^{*}\right\rangle$ and $\left\langle v_{y}^{*}\right\rangle$. These values show periodic oscillations about a geometric unit cell. Once smoothed by a second spatial average over a moving unit cell, as suggested by Barrère (1990), and substituted into the macroscale momentum equations, they allow computing $\lambda_{x}^{*}$ and $\lambda_{y}^{*}$. For the cases described above, it was found that $\lambda_{x}^{*}$ is irrelevant, i.e., $v_{\lambda x}^{*}=0$. However, the same is not true for $\lambda_{y}^{*}\left(v_{\lambda y}^{*} \neq 0\right)$. Moreover, it was noted that a subdomain made of three unit cells is sufficient as the same value of $\lambda_{y}^{*}$ was obtained on the central unit cell for a subdomain made of five (or even more) unit cells.

\subsubsection{Case a: wavy channel, $\varphi_{0}^{*}=0$}

In this case, the macroscopic pressure is constant along the system and therefore, the Darcy term plays no role in the prediction of $\left\langle\mathbf{v}^{*}\right\rangle$. In addition, $\mathbf{v}_{\lambda}^{*}=\mathbf{0}$ because $\varphi_{0}^{*}=0$ and, since $v_{d x}^{*}=0$, as already noted, $\left\langle v_{x}^{*}\right\rangle=0$. Furthermore, the vertical component of the macroscale velocity is completely determined by $v_{d y}^{*}$ (see value in Table 1 ) and, in this case, it has the value of $\left\langle v_{y}^{*}\right\rangle=-0.854$. These macroscopic results were checked to be in perfect agreement with those from the PSNS.

\subsubsection{Case b: wavy channel, $v_{0}^{*}=1$}

In this case, the mean flow goes from the centre of the system towards $\mathscr{A}_{M \beta e}$ as shown in figure $3 \mathrm{~b}$. Hence, the macroscale velocity and pressure are $x$-dependent along the channel. As shown in figure 5 , the macroscopic variables $\left\langle p^{*}\right\rangle^{\beta}$ and $\left\langle v_{y}^{*}\right\rangle$ obtained from PSNS exhibit an oscillatory behaviour, with a period corresponding to that of the geometrical 


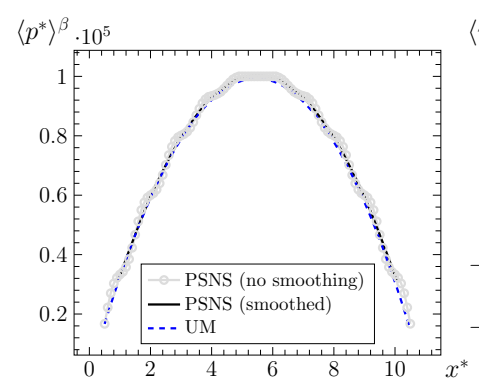

a)

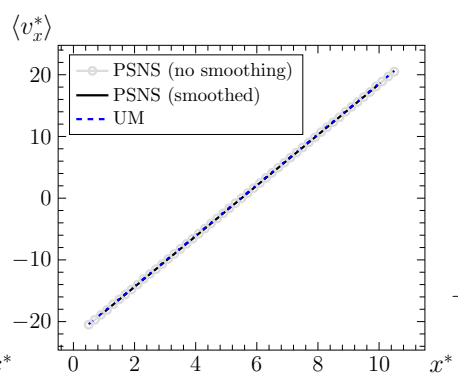

b)

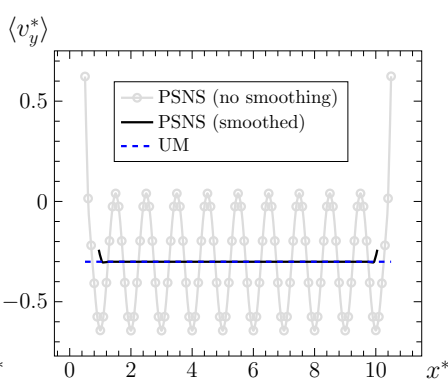

c)

Figure 5. Comparisons of the averaged results of the pore-scale numerical simulations (PSNS), with the predictions from the upscaled model (UM) for a) $\left\langle p^{*}\right\rangle^{\beta}$ b) $\left\langle v_{x}^{*}\right\rangle$, c) $\left\langle v_{y}^{*}\right\rangle$. These results correspond to Case $\mathrm{b}$ for flow in a wavy channel, in which $v_{0}^{*}=1$ at $\mathscr{A}_{\beta \sigma}$.

unit cell. The PSNS profiles can be subject to a double spatial smoothing as described above in order to eliminate the spatial oscillations. These non-oscillatory results (referred to as PSNS (smoothed) in figure 5), are in excellent agreement with the predictions from the upscaled model derived here. In this way, the macroscopic pressure follows a parabolic profile, with its maximum located at the centre of the domain that is correctly described by equation (4.7). The linear profile of the $x$-component of the macroscale velocity is in agreement with the Darcy-term contribution. For the $y$-component of the macroscopic velocity, the values of $v_{d y}^{*}$ and $v_{\lambda y}^{*}$ reported in Table 1 lead to $\left\langle v_{y}^{*}\right\rangle=-0.3$. This value matches the smoothed PSNS result as shown in figure 5c. From this point on, the doublysmoothed PSNS results are no longer reported for the sake of brevity in presentation.

\subsubsection{Case c: wavy channel, Janus-type configuration}

This case is of interest because $v_{0}^{*}$ features a heterogeneous distribution along the channel walls. Nevertheless, as expected, the global amount of exuded fluid in this case is half that in Case b, i.e., $\varphi_{0}^{*}=2.05$. Results obtained from the PSNS are qualitatively equivalent to those reported in figure $3 \mathrm{~b}$ and figure 5 , all values being only affected by a coefficient $1 / 2$. In contrast to Case $\mathrm{b},\left\langle v_{x}^{*}\right\rangle$ is shifted by $v_{d x}^{*}$, which is non-zero in the present case (see value in Table 1) due to the uneven source distribution. As indicated in Table $1, v_{d y}^{*}$ is half the value reported for Case b. In addition, the value of $\lambda_{y}^{*}$ was found to remain unaltered in comparison to Case b, so that $\left\langle v_{y}^{*}\right\rangle=-0.15$, which is half the value found in Case $\mathrm{b}$. This is again in excellent agreement with the smoothed value obtained from the PSNS and the same comment applies to the profiles of $\left\langle p^{*}\right\rangle^{\beta}$ and $\left\langle v_{x}^{*}\right\rangle$. These results are not represented here for the sake of brevity in presentation.

\subsubsection{Case d: Shark-fin channel, $v_{0}^{*}=1$}

The last channel case considered here corresponds to the shark-fin configuration with $v_{0}^{*}=1$ throughout $\mathscr{A}_{\beta \sigma}$. In contrast to Case $\mathrm{b}$, now $v_{d x}^{*}$ is non-zero due to the nonsymmetric geometry. Furthermore, taking the values of $v_{d y}^{*}$ and $v_{\lambda y}^{*}$ reported in Table 1, $\left\langle v_{y}^{*}\right\rangle=0.725$, which, again, exactly coincides with the PSNS result as shown in figure 6c. In the same figure, it is evident that $\left\langle p^{*}\right\rangle^{\beta}$ and $\left\langle v_{x}^{*}\right\rangle$ are also in agreement with the PSNS results. Contrary to the previous cases, $\left\langle p^{*}\right\rangle^{\beta}$ and $\left\langle v_{y}^{*}\right\rangle$ are not evenly distributed at the macroscopic boundaries and, interestingly, they exhibit less-pronounced oscillations along the domain than those observed in figures 5a and 5c. Finally, $\left\langle v_{x}^{*}\right\rangle$ follows a linear profile, which is not entirely described by the Darcy term since it is shifted by $v_{d x}^{*} \neq 0$. 


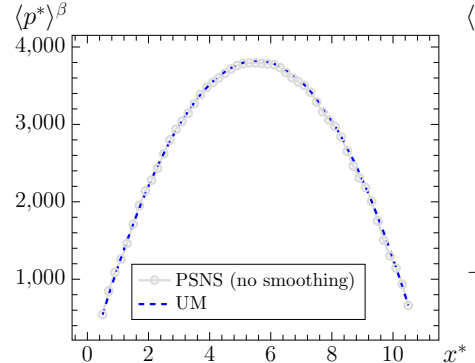

a)

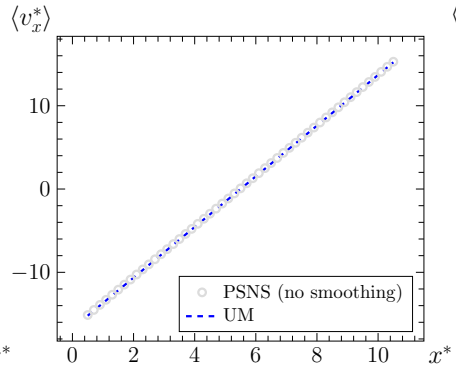

b)

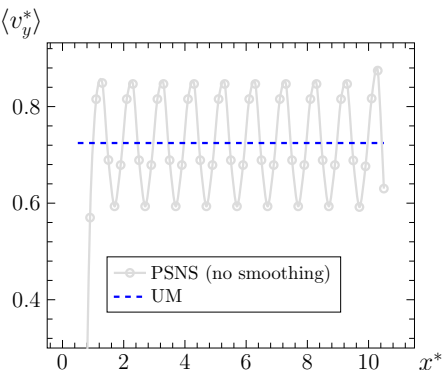

c)

FigurE 6. Comparisons of the averaged results of the pore-scale numerical simulations (PSNS), with the predictions from the upscaled model (UM) for a) $\left\langle p^{*}\right\rangle^{\beta}$ b) $\left\langle v_{x}^{*}\right\rangle$, c) $\left\langle v_{y}^{*}\right\rangle$. These results correspond to Case $\mathrm{d}$ for flow in a shark-fin channel, in which $v_{0}^{*}=1$ at $\mathscr{A}_{\beta \sigma}$.
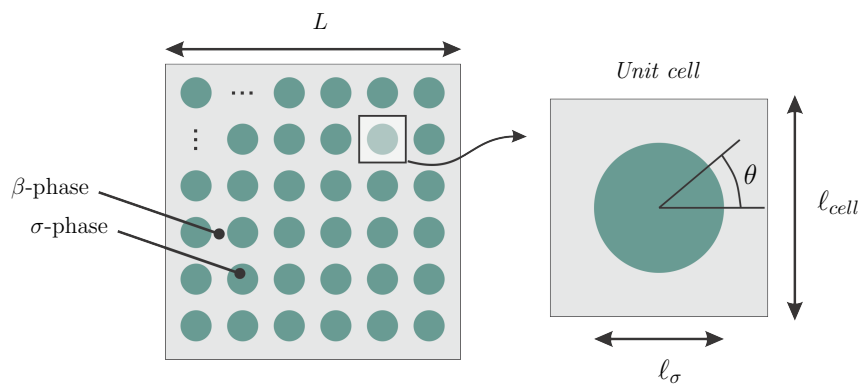

FiguRE 7. Representation of a model porous medium of characteristic length $L$, made of an array of periodic unit cells of length $\ell_{\text {cell }}$. The solid phase is modeled as a circular obstacle of diameter $\ell_{\sigma}$.

\subsection{Flow in porous media}

A second set of numerical experiments was performed in simple two-dimensional model porous media constructed by an $x$ and $y$ repetition of a periodic unit cell with isotropic and anisotropic geometries for the solid phase. Two types of distributions are considered for $v_{0}^{*}$ in the isotropic geometry:

Case a a sine-like exuding condition for which $\varphi_{0}^{*}=0$;

Case b Janus exuding grains, which refers to a single object featuring two-sided contrasted properties, namely $v_{0}^{*}=1$ and $v_{0}^{*}=0$ on each half-part of the grain surface.

The last $v_{0}^{*}$ distribution is also applied in the anisotropic geometry.

\subsubsection{Isotropic geometry}

Consider a porous medium made up of an array of periodic unit cells in which the solid phase is modelled as a circular obstacle as shown in figure 7 . The macroscopic domain is modelled as a square of side length $L=N \ell_{\text {cell }}$, with $N=11$, although similar results were observed for larger values of $N$. The porosity is taken equal to 0.8 .

\section{Case a: Sine-like exuding condition}

As a first case study, consider a situation in which $v_{0}^{*}$ obeys the following sinusoidal function

$$
v_{0}^{*}=\frac{2\left(y^{*}-y_{0}^{*}\right)}{\ell_{\sigma}^{*}}=\sin (\theta), \quad \text { at } \mathscr{A}_{\beta \sigma}
$$

where $\theta$ is the inclination of the position vector of a point on $\mathscr{A}_{\beta \sigma}$ on the horizontal axis 


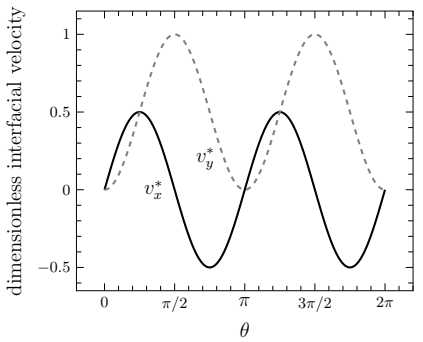

a)

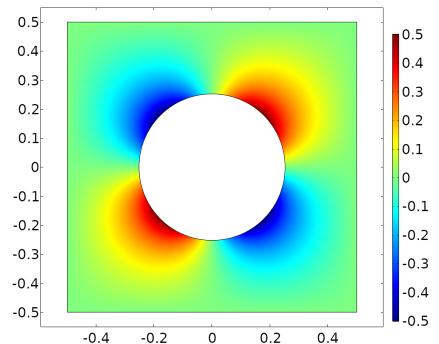

b)

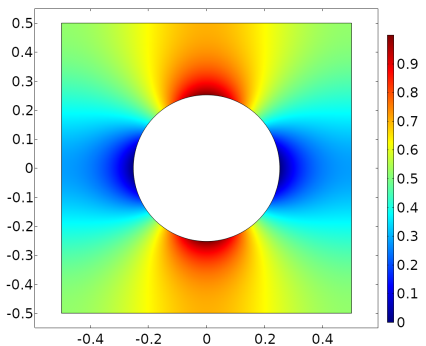

c)

Figure 8. Dimensionless pore-scale fields of a) $v_{x}^{*}=\sin (\theta) \cos (\theta)$ and $v_{y}^{*}=\sin ^{2}(\theta)$ at the solid-fluid interface corresponding to the sinusoidal distribution of $\left.v_{0}^{*}, \mathrm{~b}\right) v_{x}^{*}$ and c) $v_{y}^{*}$.

(see figure 7). In addition, $y_{0}^{*}$ is the dimensionless $y$-coordinate of the centroid of each solid inclusion, whereas $\ell_{\sigma}^{*}$ is the dimensionless diameter of the inclusion. In this way, $v_{0}^{*}$ is position-dependent at $\mathscr{A}_{\beta \sigma}$, albeit periodic. Furthermore, the boundary condition given in equation (4.9) leads to $\varphi_{0}^{*}=0$. This means that, in this case, it is physically acceptable to impose periodic boundary conditions at $\mathscr{A}_{M \beta}$ instead of equation (4.5) without compromising conservation of mass. Hence, it is not necessary to solve the porescale problem in $\mathscr{V}_{M}$ made of the $N \times N$ unit cells; it suffices to carry out the solution in only one geometrical unit cell.

The pore-scale velocity fields are presented in figure 8 both at $\mathscr{A}_{\beta \sigma}$ (figure $8 \mathrm{a}$ ) and in the unit cell domain (figures $8 \mathrm{~b}$ and $8 \mathrm{c}$ ), as a result of the PSNS. The pressure fields are not reported in this figure as they are uniformly zero. Both components of the velocity exhibit an oscillatory behaviour at $\mathscr{A}_{\beta \sigma}$; however, $v_{x}^{*}$ has cancelling amplitudes and $v_{y}^{*} \geqslant 0$. Consequently, it is not surprising that $\left\langle v_{x}^{*}\right\rangle=0$; in addition, $\left\langle v_{y}^{*}\right\rangle=0.404$.

Focusing now on the upscaled model, the closure problem was solved in the periodic unit cell sketched in figure 7 . The resulting fields of the closure variables are represented in figure 9 . Clearly, the diagonal components of tensor $\mathbf{D}^{*}$ are $x$ and $y$-symmetric fields, whereas the off-diagonal components are antisymmetric. As a consequence, $\mathbf{K}^{*}$ is a spherical tensor, i.e., $\mathbf{K}^{*}=K^{*} \mathbf{I}$ and the value of $K^{*}$ is reported in Table 1 .

Regarding the fields of the closure vector $\mathbf{d}_{1}^{*}$, the results for the $x$ - and $y$-components are represented in figures $9 \mathrm{e}$ and $9 \mathrm{f}$ showing that $d_{1 x}$ and $d_{1 y}$ are antisymmetric about the vertical and horizontal directions, respectively. It is worth recalling that these results are intrinsic to the geometry i.e., they are independent of $v_{0}^{*}$. In addition, since there is no macroscopic pressure gradient in any direction, it follows that the Darcy term in the macroscale momentum equation $(3.20 b)$ is zero, thus simplifying the model to $\left\langle\mathbf{v}^{*}\right\rangle=\mathbf{v}_{s}^{*}$. Finally, since $\varphi_{0}^{*}=0$ in this case, $\mathbf{v}_{\lambda}^{*}=\mathbf{0}$ and $\mathbf{v}_{s}^{*}=\mathbf{v}_{d}^{*}$. With the values reported in Table 1 , this leads to a perfect match with the PSNS data.

Case b: Janus exuding grains

The second case study deals with a Janus-type configuration for $v_{0}^{*}$, such that

$$
v_{0}^{*}=\left\{\begin{array}{lll}
1 & \text { when } \quad 0<\theta<\pi / 2 \text { and } 3 \pi / 2<\theta<2 \pi \\
0 & \text { when } \pi / 2<\theta<3 \pi / 2
\end{array}\right.
$$

Solving the governing equations at the pore-scale subject to the macroscopic boundary condition provided in equation (4.5) gives rise to the velocity and pressure fields reported in figure 10. The field of $v_{x}^{*}$ is $y$-symmetric but not $x$-symmetric, whereas the field of $v_{y}^{*}$ is $x$-symmetric and $y$-antisymmetric. The pressure field is $x$ and $y$-symmetric with a maximum value located at the centre of the domain. 


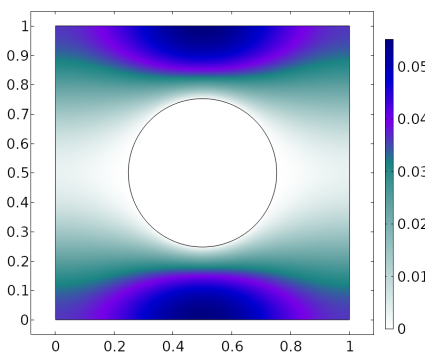

a)

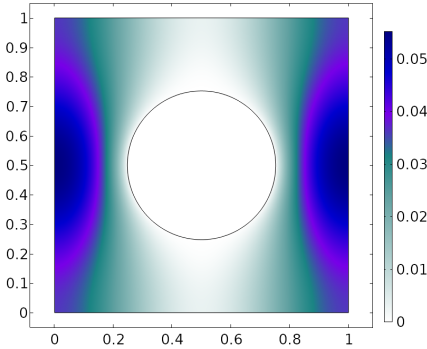

d)

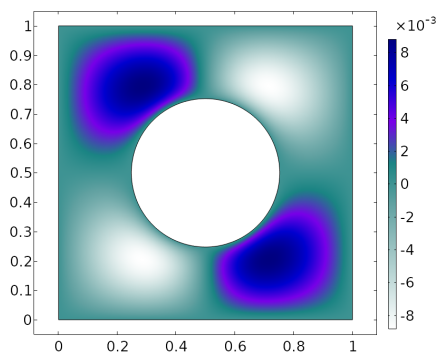

b)

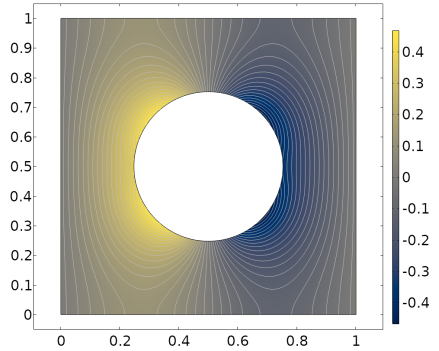

e)

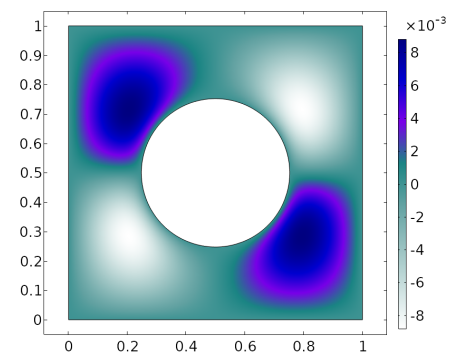

c)

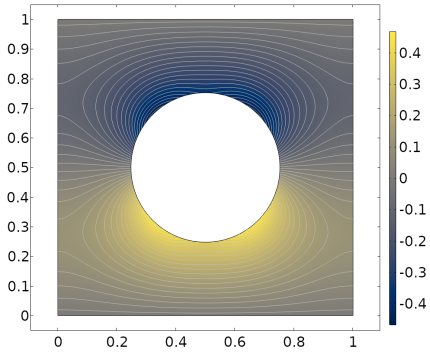

f)

Figure 9. Fields of the closure variables a) $D_{x x}^{*}$, b) $D_{y x}^{*}$, c) $D_{x y}^{*}$, d) $D_{y y}^{*}$, e) $d_{1 x}^{*}$, f) $d_{1 y}^{*}$ in the periodic unit cell sketched in figure 7 for a porosity value of 0.8 .

$\begin{array}{lllllll}-6 & -4 & -2 & 0 & 2 & 4 & 6\end{array}$

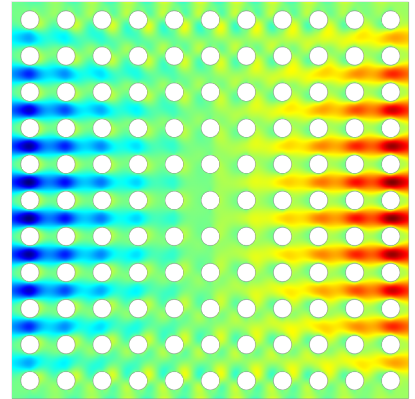

a)

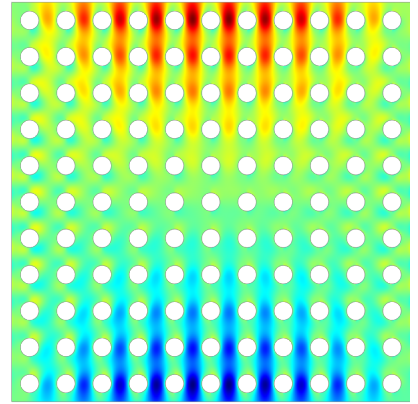

b)

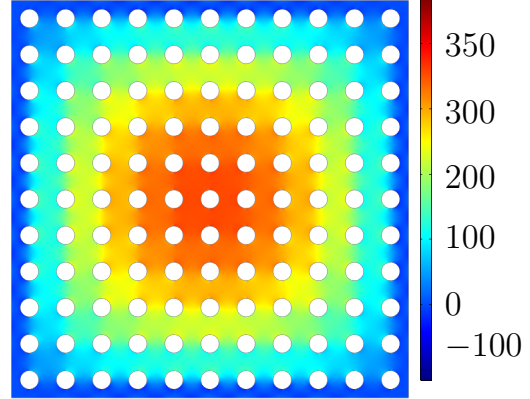

c)

Figure 10. Fields of a) $v_{x}^{*}$, b) $v_{y}^{*}$, c) $p^{*}$ obtained from pore-scale numerical simulations considering the Janus exuding grains.

These observations suggest that the solution of the governing macroscale pressure differential equation

$$
\nabla^{* 2}\left\langle p^{*}\right\rangle^{\beta}=-\frac{\varphi_{0}^{*}}{K^{*}}
$$

can be performed in one quarter of the macroscopic domain. Notice that, in the above equation, the isotropic nature of the permeability tensor was taken into account. Locating the coordinate axes origin at the macroscopic domain centre, the solution of equation 


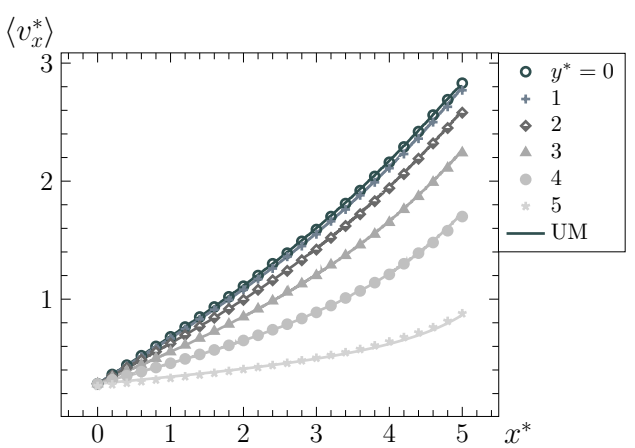

a)

$\left\langle v_{y}^{*}\right\rangle$

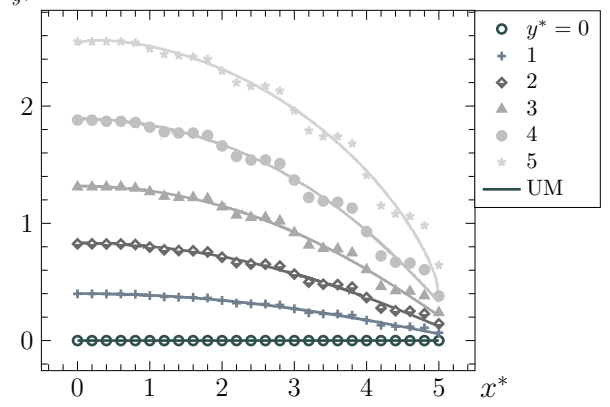

c)

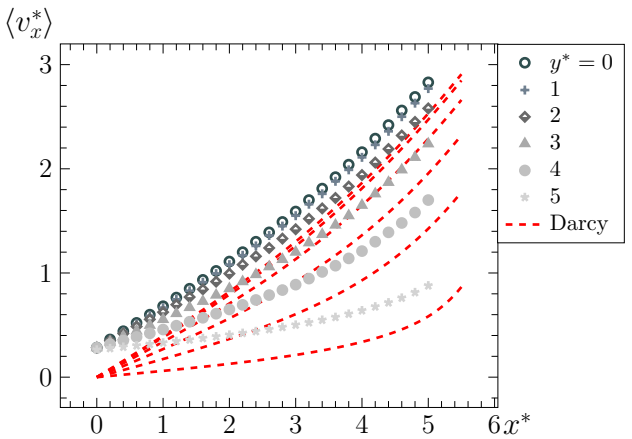

b)

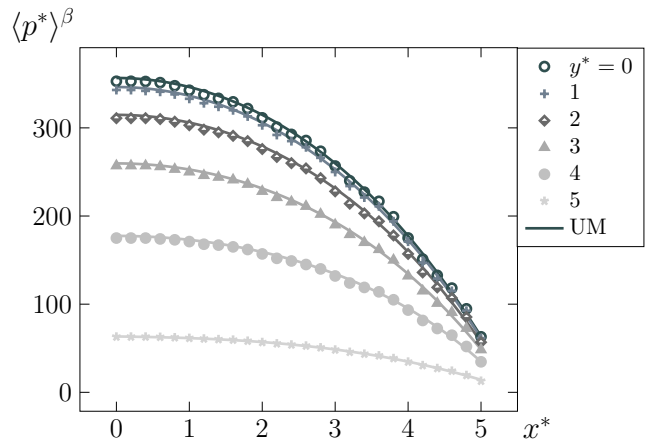

d)

Figure 11. Comparison of the macroscopic velocity ( $a, b$ and $c$ ) and pressure (d) profiles obtained from the upscaled model (UM) and pore-scale numerical simulations (PSNS) for the Janus exuding grains. In b), the horizontal averaged velocity results from PSNS are compared with those from Darcy's law.

(4.11a) is subject to the following set of boundary conditions

$$
\begin{array}{ll}
\text { at } x^{*}=0, & \frac{\partial\left\langle p^{*}\right\rangle^{\beta}}{\partial x^{*}}=0 \\
\text { at } y^{*}=0, & \frac{\partial\left\langle p^{*}\right\rangle^{\beta}}{\partial y^{*}}=0 \\
\text { at } x^{*}=x_{L}^{*}, & \left\langle p^{*}\right\rangle^{\beta}=p_{L x}^{*}\left(y^{*}\right) \\
\text { at } y^{*}=y_{L}^{*}, & \left\langle p^{*}\right\rangle^{\beta}=p_{L y}^{*}\left(x^{*}\right)
\end{array}
$$

The first two boundary conditions follow from the macroscale pressure-field symmetries. As in the analysis of the corrugated channels, $x_{L}^{*}$ and $y_{L}^{*}$ locate the positions of the last centroids of the unit cells where the PSNS data were collected (i.e., $x^{*}=y^{*}=5$ ). Hence, the functions $p_{L x}^{*}\left(y^{*}\right)$ and $p_{L y}^{*}\left(x^{*}\right)$ are extracted from the PSNS.

Due to the $y$-antisymmetry of $d_{1 y}^{*}$ exhibited in figure 9 f, it follows that $v_{d y}^{*}=0$. With the data reported in Table 1 for $v_{d x}^{*}$ and $\varphi_{0}^{*}$, it is possible to carry out the upscaled model solution and compute the profiles of the macroscale velocity and pressure. These results are reported in figure 11 and they are compared with those arising from the average PSNS data for various values of $y^{*}$. The simulations from the upscaled model are found to be in good agreement with the PSNS without requiring the computation of $\mathbf{v}_{\lambda}^{*}$. In order to appreciate the relevance of $\mathbf{v}_{d}^{*}$, the predictions of $\left\langle v_{x}^{*}\right\rangle$, only considering the Darcy term, are compared with the PSNS results in figure 11b, showing a clear discrepancy. The two 


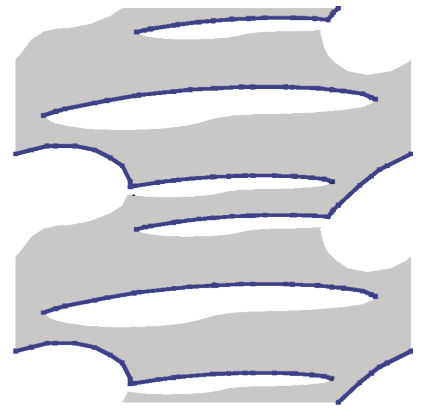

Figure 12. Periodic unit cell used to construct the anisotropic porous medium. The boundaries at which $v_{0}^{*}$ is set to one are shaded in blue.

sets of curves differ by a constant vertical shift, corresponding to $v_{d x}^{*}$. It should be noted that the magnitude of the macroscopic pressure gradient builds up while moving away from the centre of the domain, thus increasing $\left\langle v_{x}^{*}\right\rangle$. Consequently, since $\mathbf{v}_{d}^{*}$ is a constant vector, its relative contribution decreases when the Darcy term becomes dominant.

When considering the results on $\left\langle v_{y}^{*}\right\rangle$ and $\left\langle p^{*}\right\rangle^{\beta}$ represented in figure $11 \mathrm{c}$ and $11 \mathrm{~d}$, respectively, it is observed that the averaged PSNS results follow an oscillatory behaviour, more prominent for $\left\langle v_{y}^{*}\right\rangle$. Nevertheless, the predictions from the upscaled model are in excellent agreement with the PSNS results once doubly-smoothed for all the values of $y^{*}$ considered here.

\subsubsection{Anisotropic geometry}

To conclude this section, consider now flow in an anisotropic medium with porosity $\varepsilon=0.621$, constructed by a $N \times N$ repetition in the $x$ and $y$ directions $(N=11$ in the present case) of the periodic unit cell shown in figure 12 . For this case, $v_{0}^{*}=1$ at the blue shaded part of $\mathscr{A}_{\beta \sigma}$ shown in this figure, while $v_{0}^{*}=0$ at the remaining part of the solid-fluid interfaces. This arrangement is referred to as a vertical Janus configuration in the following paragraphs. Imposing the boundary condition given in equation (4.5) at $\mathscr{A}_{M \beta e}$, and carrying out PSNS, yields the velocity and pressure fields shown in figure 13 . The numerical results are somewhat similar to those presented in figure 11 in the sense that the fluid tends to move from the centre towards the macroscopic boundaries.

The fields of all the closure variables, obtained after solving the closure problem in the geometric unit cell shown in figure 12, are represented in figure 14. Clearly, no symmetric or antisymmetric property is observed. From this solution, the components of the permeability tensor, which is now a full matrix, can be computed and the results are reported in Table 1 along with the values of $\varphi_{0}^{*}, v_{d x}^{*}$ and $v_{d y}^{*}$.

Since no symmetry properties are applicable to the pressure, the upscaled model must be solved in the entire macroscopic domain, $\mathscr{V}_{M}$. Locating the origin of the coordinate axes at the system centre, the macroscale pressure solves the following boundary-value problem

$$
\begin{aligned}
& \mathbf{K}^{*}: \nabla^{*} \nabla^{*}\left\langle p^{*}\right\rangle^{\beta}=-\varphi_{0}^{*} \\
& \text { at } x^{*}=-x_{L}^{*}, \quad\left\langle p^{*}\right\rangle^{\beta}=p_{0 x}^{*}\left(y^{*}\right) \\
& \text { at } x^{*}=x_{L}^{*}, \quad\left\langle p^{*}\right\rangle^{\beta}=p_{L x}^{*}\left(y^{*}\right) \\
& \text { at } y^{*}=-y_{L}^{*}, \quad\left\langle p^{*}\right\rangle^{\beta}=p_{0 y}^{*}\left(x^{*}\right) \\
& \text { at } y^{*}=y_{L}^{*}, \quad\left\langle p^{*}\right\rangle^{\beta}=p_{L y}^{*}\left(x^{*}\right)
\end{aligned}
$$




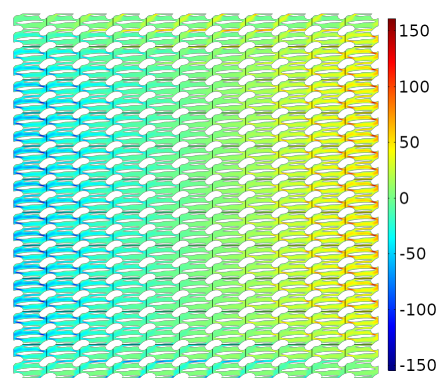

a)

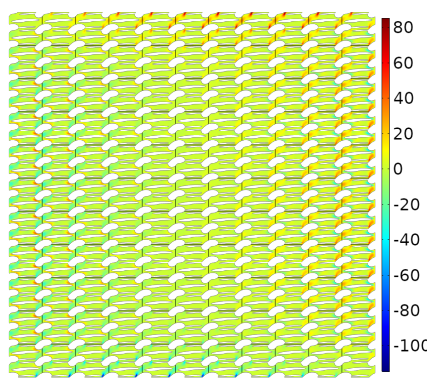

b)

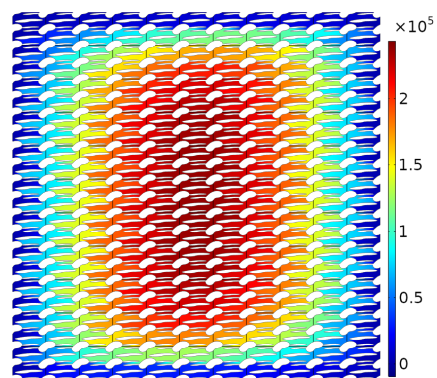

c)

Figure 13. Pore-scale fields of a) $v_{x}^{*}$, b) $v_{y}^{*}$ and c) $p^{*}$ obtained from the vertical Janus configuration in the anisotropic geometry.

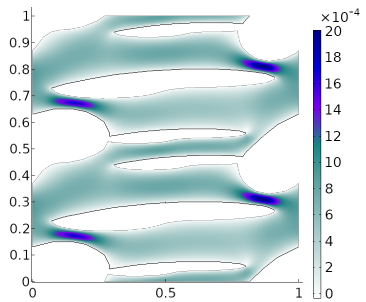

a)

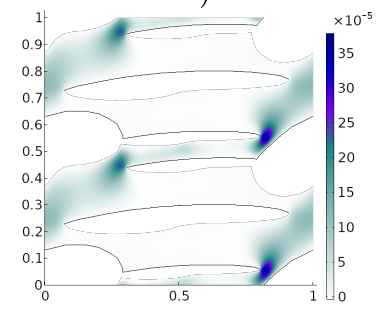

d)

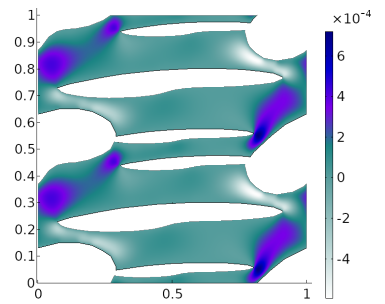

b)

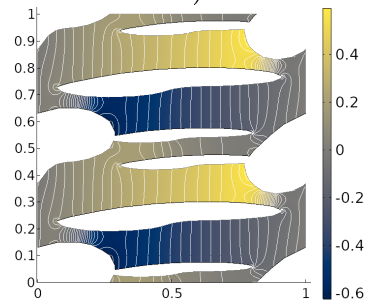

e)

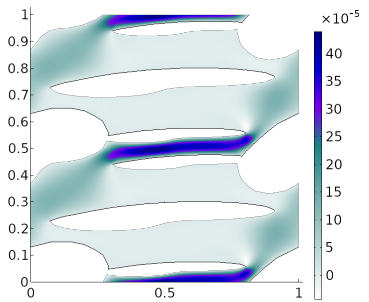

c)

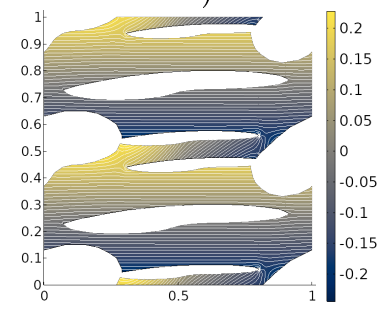

f)

Figure 14. Fields of the closure variables a) $D_{x x}^{*}$, b) $D_{y x}^{*}$, c) $D_{x y}^{*}$, d) $D_{y y}^{*}$, e) $d_{x}^{*}$, f) $d_{y}^{*}$ for the anisotropic geometry.

As in the previous case, $x_{L}^{*}=y_{L}^{*}=5$, while $p_{0 i}^{*}$ and $p_{L i}^{*}(i=x, y)$ are functions of position that are obtained from the PSNS. Despite the fact that this problem was solved in the entire domain, the comparisons with the PSNS data are reported in figure 15 for only one quarter of $\mathscr{V}_{M}$ for the sake of brevity in presentation. The macroscale velocity and pressure profiles barely change for $y^{*}=0,1$ and 2 and for this reason, the results corresponding to $y^{*}=1$ and $y^{*}=2$ are not reported in this figure. These results are in excellent agreement with the predictions from the upscaled model without considering the contribution from $\mathbf{v}_{\lambda}^{*}$. Note that, as in Case a for the isotropic porous medium, the relative importance of $\mathbf{v}_{d}^{*}$ with respect to the $\left\langle\mathbf{v}^{*}\right\rangle$ decreases while moving away from the centre of the domain.

Finally, it is worth considering another distribution of $v_{0}^{*}$ in the same porous medium, which can be viewed as a horizontal Janus exuding pattern. As for the vertical Janus distribution of $v_{0}^{*}$, an excellent agreement is found between the averaged PSNS results and the solution of the upscaled model, without taking $\mathbf{v}_{\lambda}^{*}$ into account that is again irrelevant in that case. The values of $\varphi_{0}^{*}$ and $\mathbf{v}_{d}^{*}$ are given in Table 1 . Most interestingly, if the contribution from $\mathbf{v}_{s}^{*}$ is neglected and if the results are normalized by $\varphi_{0}^{*}$, then the macroscale velocity and pressure profiles from the vertical Janus match those from the 


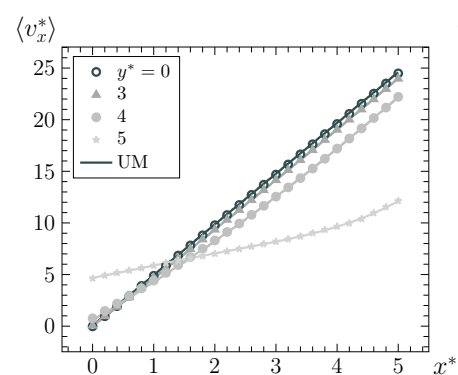

a) $\left\langle v_{y}^{*}\right\rangle$

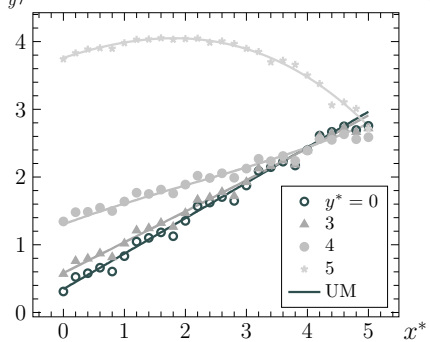

b)

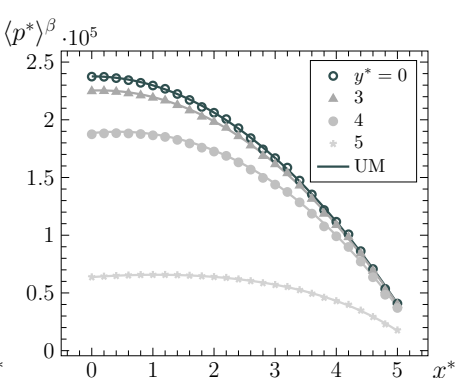

c)

Figure 15. Comparison of the macroscopic velocity (a and b) and pressure (c) obtained from the upscaled model (UM) and the pore-scale numerical simulations (PSNS) for the vertical Janus configuration in the anisotropic medium.

horizontal Janus. This is expected, since when the net volume sources and outer boundary conditions are identical, the macroscopic model (3.20) predicts that the velocity fields differ only by the value of $\mathbf{v}_{s}^{*}$ associated with a particular distribution of the interfacial sources. This is a relevant result, that may be of interest in practical situations in which one may only account for $\varphi_{0}^{*}$ at the macroscale.

\subsection{Migration of water vapor bubbles in ice}

To conclude this section, an extension of this work to the migration of water vapour bubbles in ice is briefly discussed. To this end, consider an ice mass containing a dilute population of spherical bubbles. For simplicity, assume that the bubbles contain pure water vapour, so that internal mass transfer does not involve diffusion. Each bubble is supposed to be in global equilibrium, i.e., its internal pressure is equal to the saturation pressure at the temperature of the surrounding ice and its volume is constant. Any deviation would result in a net ice sublimation or vapor condensation rate, with pressure and volume variations, until an equilibrium state is reached ultimately.

Despite the global equilibrium, an overall temperature gradient can exist in the ice mass, as occurs in glaciers or polar ice caps (Mellor 1960). The resulting slight temperature difference across the bubble causes sublimation to take place at the warm part of its surface, and condensation on the opposite cold side. This, in turn, induces a migration of the bubble in the direction of increasing temperature. Under a quasi-steady approximation, it is reasonable to neglect interfacial displacement, so that, the transfers at a bubble boundary correspond to

$$
v_{0}^{*}=\frac{\rho_{i}}{\rho_{v}} \mathbf{n} \cdot \underbrace{\left(\frac{3 k_{i}}{\rho_{i} L_{s} v_{r e f}} \nabla T\right)}_{\mathbf{v}_{B}^{*}}, \quad \text { at } \mathscr{A}_{\beta \sigma}
$$

Here $\rho_{i}$ and $\rho_{v}$ denote the densities of ice and water vapour, respectively. In addition, $k_{i}$ is the ice thermal conductivity, $L_{s}$ is the latent heat of sublimation and $\nabla T$ is the overall temperature gradient. Finally, the term between parenthesis in the above equation corresponds to the dimensionless bubble velocity, $\mathbf{v}_{B}^{*}$. Its expression results from an energy balance, and shows that it does not depend on the bubble size (Shreve 1967). The source distribution in equation (4.13) results in $\varphi_{0}^{*}=0$, in agreement with the global equilibrium hypothesis.

A simple way to derive an expression for the macroscopic vapor velocity, without actually solving the flow problem, is by noticing that $\mathbf{v}^{*}=\nabla^{*} \cdot\left(\mathbf{v}^{*} \mathbf{r}^{*}\right)$ and applying the 
TABlE 2. Summary of the macroscopic velocity features in the different case studies. The values of $v_{d x}^{*}, v_{d y}^{*}$ and $v_{\lambda y}^{*}$ are reported in Table 1 .

\begin{tabular}{lccc}
\hline \multicolumn{1}{c}{ Case } & $\varphi_{0}^{*}$ & $\left\langle v_{x}^{*}\right\rangle$ & $\left\langle v_{y}^{*}\right\rangle$ \\
\hline Flat channel & $\varepsilon\left(v_{0}^{+*}+v_{0}^{-*}\right)$ & Darcy & $v_{d y}^{*}$ \\
Wavy channel Case a & 0 & 0 & $v_{d y}^{*}$ \\
Wavy channel Case b & 4.11 & Darcy & $v_{d y}^{*}+v_{\lambda y}^{*}$ \\
Wavy channel Case c & 2.05 & Darcy $+v_{d x}^{*}$ & $v_{d y}^{*}+v_{\lambda y}^{*}$ \\
Shark-fin channel Case d & 3.04 & Darcy $+v_{d x}^{*}$ & $v_{d y}^{*}+v_{\lambda y}^{*}$ \\
Isotropic porous medium Case a & 0 & 0 & $v_{d y}^{*}$ \\
Isotropic porous medium Case b & 0.793 & Darcy $+v_{d x}^{*}$ & Darcy \\
Anisotropic porous medium & 4.92 & Darcy $+v_{d x}^{*}$ & Darcy $+v_{d y}^{*}$ \\
vertical Janus & 4.81 & Darcy $+v_{d x}^{*}$ & Darcy $+v_{d y}^{*}$ \\
Anisotropic porous medium & 0 & \multicolumn{2}{c}{$\mathbf{v}_{d}^{*} / / \nabla T$} \\
horizontal Janus & \multicolumn{2}{c}{} \\
Bubble & \multicolumn{2}{c}{} \\
\hline
\end{tabular}

${ }^{\dagger}$ The term Darcy denotes the projection of $-\mathbf{K}^{*} \cdot \nabla^{*}\left\langle p^{*}\right\rangle^{\beta}$ on the corresponding direction.

intrinsic averaging operator together with the divergence theorem to obtain

$$
\left\langle\mathbf{v}^{*}\right\rangle^{\beta}=-\frac{1}{V_{\beta}^{*}} \int_{\mathscr{A}_{\beta \sigma}} v_{0}^{*} \mathbf{r}^{*} d A^{*}=-\frac{\rho_{i}}{\rho_{v}} \mathbf{v}_{B}^{*}
$$

The last equality results from substitution of $v_{0}^{*}$ from equation (4.13) in the integral. In fact, this interfacial integral can be identified as the source dipole. For instance, if the source and sinks were concentrated at two points, it would reduce to a usual point-source dipole (Pozrikidis 2003; Spagnolie \& Lauga 2012).

Since the pore space in the ice is made of disconnected cavities, its permeability coefficient is zero, hence the Darcy-term in equation (3.16) vanishes and the macroscopic velocity reduces to $\left\langle\mathbf{v}^{*}\right\rangle=\mathbf{v}_{s}^{*}=\mathbf{v}_{d}^{*}$ because $\varphi_{0}^{*}=0$. In this way, the closure problem given in equations (3.14) can be solved in a unit cell containing a single bubble. It is readily apparent that $\mathbf{D}^{*}=\mathbf{0}$ (and therefore $\mathbf{K}^{*}=\mathbf{0}$ ) and $\mathbf{d}_{1}^{*}=\mathbf{r}^{*}-\mathbf{r}_{G}^{*}$ as mentioned in section 3.2 (see equations (3.19)). Substitution of this result, along with the expression of $v_{0}^{*}$ given in equation (4.13), into equation (3.17), taking into account the fact that $\varphi_{0}^{*}=0$, leads to an expression for $\left\langle\mathbf{v}^{*}\right\rangle$, which, when divided by the porosity, allows recovering the result given in equation (4.14). This can be straightforwardly extended to periodic media where the unit cell contains any number of bubbles, possibly with different sizes. Thus, the upscaled model established in the previous section accommodates this peculiar situation of a non-percolating porous medium, and predicts the correct mean gas velocity.

To summarise the different situations investigated with the above examples, the features of the macroscopic velocity components are reported in Table 2, highlighting the richness of the problem. Note that the Darcy model is applicable in the minority of the cases studied here. When $\varphi_{0}^{*}=0$, there is no Darcy-term in the macroscale model. 


\section{Discussion and conclusions}

In this work, a macroscale model describing incompressible, Newtonian and creeping flow in exuding rigid and homogeneous porous media was derived. The governing transport equations at the pore-scale correspond to the ones involved in the derivation of Darcy's law with the difference that a normal mass flux is present at the solid-fluid interface. Despite the apparent simplicity of this problem, this physical situation is quite rich. Indeed, mass conservation prevents, in general, the simplification of the problem to a single periodic unit cell. Therefore, the use of periodic boundary conditions in the course of the upscaling process must be treated with caution. Nevertheless, a common practice in the literature is to use the classical Darcy's law at the macroscopic level, which raises the question about the pertinence of such an equation and/or whether or not the permeability tensor can remain intrinsic. The upscaling of the governing pore-scale equations developed in this work contrasts to the Darcy model in two respects. First, the macroscopic velocity field is non-solenoidal. The non-solenoidal nature of the average velocity (despite the fact that the flow is incompressible) is a relevant modification to the classical Darcy model, albeit not the only one, as it accounts for the mass entering/exiting the system through the solid-fluid interface. Second, the macroscale momentum equation involves the classical Darcy term (with the intrinsic permeability) and a correction term. The correction term, $\mathbf{v}_{s}$, in the macroscopic momentum equation is the sum of a contribution, $\mathbf{v}_{d}$, due to the local effect of $v_{0}$ and a term, $\mathbf{v}_{\lambda}$, that compensates for the periodicity assumption. The magnitude of both $\mathbf{v}_{d}$ and $\mathbf{v}_{\lambda}$ were found to be, at most, of the order of $v_{0}$. Since $\mathbf{v}_{s}$ is a vector, the correction only applies in one spatial direction. This can only occur if the system features privileged directions that result from geometrical and/or $v_{0}$ distribution anisotropy. A salient feature of the model derived here is that a unique intrinsic closure problem, exactly corresponding to the one involved in the classical Darcy case, is required to predict both the intrinsic permeability tensor and $\mathbf{v}_{d}$. The correction term, $\mathbf{v}_{s}$, accounts for the local fluid displacement induced by the exuding phenomenon at the solid-fluid interfaces. This effect is not captured by the Darcy term, which only accounts for the macroscopic drag. This correction term, $\mathbf{v}_{s}$, identifies as the average of a contribution to the flow field which does not induce any macroscopic force on the solid skeleton of the porous material.

The performance of the upscaled model was validated in a variety of physical situations ranging from flow in flat and corrugated channels to isotropic and anisotropic porous media. For channels, the component along the channel-axis of the correction term in the macroscopic momentum equation results from a complex interplay of the distribution of $v_{0}$ and the walls geometry. This component is zero in the case of the plane channel (it can be shown that this result holds whatever $v_{0}$ ) or in the sine-channel when $v_{0}$ is uniform. In more complex cases, a correction is needed in the axial direction and it can only be obtained from the solution of the adjoint problem. The different channel examples illustrate the pertinence of the correction in various flow situations; a detailed analysis on the role played by the topology and $v_{0}$ distribution is out of the scope of this work.

The macroscale model solution requires physically sound boundary conditions at the macroscopic boundaries. In this work, they were obtained by fitting data from the porescale numerical simulations at the macroscopic boundaries. Certainly, it is tempting to impose heuristic boundary conditions, such as $\langle p\rangle^{\beta}=0$ at $\mathscr{A}_{M \beta e}$. However, it was verified that using this approach leads to relative percent errors larger than $10 \%$, especially near the macroscopic boundaries. In contrast, the approach used here leads to an error below $1 \%$. Certainly, this issue requires further analysis since the derivation of macroscopic 
boundary conditions is still a developing research subject not specific to the exuding problem investigated here. However, this is beyond the scope of the current work.

For the cases in which $\varphi_{0}=0$ (plane channel when $\varphi_{0}=0$, wavy channel Case a, isotropic porous medium Case a, and bubbles in ice), periodicity is physically justified and the macroscopic model is exact to within the approximation associated to the classical length-scale constraint. Since no macroscopic pressure gradient is present, it follows that a Darcy-type model is incapable of predicting the constant velocity. This result is accurately predicted by $\mathbf{v}_{d}$ with $\mathbf{v}_{\lambda}=\mathbf{0}$.

When the distribution of $v_{0}$ is such that $\varphi_{0} \neq 0$, many different situations can occur. The pertinence of the constant correction term $\mathbf{v}_{d}$ with respect to the Darcy term is particular to each application. Moreover, the correction term $\mathbf{v}_{\lambda}$ was found to be generally irrelevant for the cases studied here. The exception is in the corrugated channel cases, for which $\mathbf{v}_{\lambda}$ plays a role in the non-percolating direction of the channel.

The above indicates that a Darcy-type model may be pertinent whenever the pressure gradient spatially develops so that the Darcy term overcomes the contribution from $\mathbf{v}_{s}$. Nevertheless, it should be noticed that, even if a Darcy model would provide acceptable predictions of the macroscopic velocity, in some particular situations, $\mathbf{v}_{s}$ may not be neglected (see Table 2). Indeed, it can trigger some mechanisms, like instability for instance, if flow is coupled to another non-linear phenomenon, which may be overlooked if a pure Darcy model is employed.

As a matter of perspective, the combination of the upscaled model from this work with species and/or heat transport models can be used to predict an ample variety of transport processes in porous media involving internal flow sources, such as mineral dissolution in rough fractures as recently studied by Rasoulzadeh et al. (2020). Finally, the results from this work should also call for complementary experimental investigation.

\section{Acknowledgements}

D.L. would like to gratefully acknowledge support from UAM (CDMX) in November 2018 and 2019, a period during which part of this work was completed. F.J.V.-P. would like to thank the CNRS for the support for a one-month stay at I2M during 2019. D.L. and F.J.V.-P. are most thankful to A. Bottaro (University of Genoa) for insightful discussions.

\section{Appendix A. Order of magnitude estimates}

The purpose of this appendix is to provide order of magnitude estimates for the correction vectors $\mathbf{v}_{d}$ and $\mathbf{v}_{\lambda}$. To this end, it is convenient to commence the analysis by examining the boundary-value problem given in equations (3.3), which are subject to homogeneous boundary conditions at $\mathscr{A}_{\beta}$. This means that this problem is driven by the unitary volume source in equation $(3.3 b)$. From this observation, the following order of magnitude estimate can be made

$$
\mathbf{d}=\mathbf{O}\left(\ell_{\beta}\right)
$$

Since $\boldsymbol{\lambda}=\langle\mathbf{d}\rangle^{\beta}$, it follows that $\boldsymbol{\lambda}=\mathbf{O}\left(\ell_{\beta}\right)$, with the idea that this represents an upper bound. Directing the attention to the closure problem defined in equations (3.14), a similar analysis leads to the estimate

$$
\mathbf{d}_{1}=\mathbf{O}\left(\ell_{\beta}\right)
$$


Taking these results into account, the order of magnitude estimates for $\mathbf{v}_{d}$ and $\mathbf{v}_{\lambda}$ are derived

$$
\begin{aligned}
& \mathbf{v}_{d}=-\frac{1}{V} \int_{\mathscr{A}_{\beta \sigma}} v_{0} \mathbf{d}_{1} d A=\mathbf{O}\left(v_{0}\right) \\
& \mathbf{v}_{\lambda}=-\boldsymbol{\lambda} \varphi_{0}=\mathbf{O}\left(v_{0}\right)
\end{aligned}
$$

which are again upper bounds. With these results, it immediately follows that $\mathbf{v}_{s}=\mathbf{O}\left(v_{0}\right)$ and this concludes the derivations.

\section{Declaration of interests}

The authors report no conflict of interest.

\section{REFERENCES}

Adler, Pierre 1992 Porous Media: Geometry and Transports. Butterworth-Heinemann Series in Chemical Engineering.

Barrère, J. 1990 Modélisation des écoulement de Stokes et de Navier-Stokes en milieu poreux. $\mathrm{PhD}$ thesis, University of Bordeaux.

Bottaro, A. 2019 Flow over natural or engineered surfaces: an adjoint homogenization perspective. Journal of Fluid Mechanics 877, 1-91.

Bryden, K. M., Ragland, K. W. \& Rutland, C. J. 2002 Modeling thermally thick pyrolysis of wood. Biomass and Bioenergy 22, 41-53.

Dadic, R., Light, B. \& Warren, S. G. 2010 Migration of air bubbles in ice under a temperature gradient, with application to "snowball earth". Journal of Geophysical Research 115, D18125.

Davit, Y., Bell, C. G., Byrne, H. M., Chapman, L. A. C., Kimpton, L. S., Lang, G. E., Leonard, K. H. L., Oliver, J. M., Pearson, N. C., Shipley, R. J., Waters, S. L., Whiteley, J. P., Wood, B. D. \& Quintard, M. 2013 Homogenization via formal multiscale asymptotics and volume averaging: How do the two techniques compare? Advances in Water Resources 62, 178-206.

Di Blasi, C. 1994 Numerical simulation of cellulose pyrolysis. Biomass and Bioenergy 7, 87-98.

Elayeb, M., Debenest, G., Mourzenko, V. \& Thovert, J.-F. 2017 Smoldering combustion in oil shales: Influence of calcination and pyrolytic reactions. Transport in Porous Media 116, 889-921.

Erriguible, A., Bernada, P., Couture, F. \& Roques, M. 2006 Simulation of convective drying of a porous medium with boundary conditions provided by CFD. Chemical Engineering Research and Design 84, 113-123.

Flin, F., Brzoska, J.-B., Lesaffre, B., Coléou, C. \& Pieritz, R. A. 2003 Full threedimensional modelling of curvature-dependent snow metamorphism: first results and comparison with experimental tomographic data. Journal of Physics D: Applied Physics 36, A49-A54.

Gray, W. G. 1975 A derivation of the equations for multi-phase transport. Chemical Engineering Science 30 (2), 229-233.

JiAnG, J. \& Younis, R. M. 2015 Numerical study of complex fracture geometries for unconventional gas reservoirs using a discrete fracture-matrix model. Journal of Natural Gas Science and Engineering 26, 1174 - 1186.

Lachaud, J., Scoggins, J. B., Magin, T. E., Meyer, M. G. \& Mansour, N. N. 2017 A generic local thermal equilibrium model for porous reactive materials submitted to high temperatures. International Journal of Heat and Mass Transfer 108, 1406-1417.

Larfeldt, J., Leckner, B. \& Melaaen, M. C. 2000 Modelling and measurements of the pyrolysis of large wood particles. Fuel 79, 1637-1643.

LAsSEux, D. \& VAldÉs-Parada, F. J. 2017 Symmetry properties of macroscopic transport coefficients in porous media. Physics of Fluids 29 (4), 043303. 
Lasseux, D., Valdés-Parada, F. J. \& Porter, M. L. 2016 An improved macroscale model for gas slip flow in porous media. Journal of Fluid Mechanics 805, 118-146.

Mahmoudi, A. H., Hoffmann, F. \& Peters, B. 2014 Detailed numerical modeling of pyrolysis in a heterogeneous packed bed using XDEM. Journal of Analytical and Applied Pyrolysis 106, 9-20.

Mei, C. C., Auriault, J.-L. \& NG, C.-O. 1996 Some applications of the homogenization theory. In Advances in Applied Mechanics, pp. 277-348. Elsevier.

Mei, C. C. \& Vernescu, B. 2010 Homogenization Methods For Multiscale Mechanics. World Scientific Publishing Company.

Mellor, M. 1960 Temperature gradients in the Antarctic ice sheet. Journal of Glaciology 28 (3), 773-782.

Mourzenko, V., Thovert, J.-F. \& Adler, P.M. 2018 Conductivity and transmissivity of a single fracture. Transport in Porous Media 123, 235-256.

Olorode, O. M., Freeman, C. M., Moridis, G. J. \& Blasingame, T. 2012 High-resolution numerical modeling of complex and irregular fracture patterns in shale gas and tight gas reservoirs. SPE Reservoir Evaluation \& Engineering 6, SPE-152482-MS.

Pozrikidis, C. 2003 Computation of the pressure inside bubbles and pores in Stokes flow. Journal of Fluid Mechanics 474, 319-337.

Rasoulzadeh, M., Yekta, A., Deng, H. \& Ghahfarokhi, R. B. 2020 Semi-analytical models of mineral dissolution in rough fractures with permeable walls. Physics of Fluids 32 (5), 052003.

Rönsch, S., Schneider, J., Matthischie, S., Schlüter, M., Götz, M., Lefebvre, J., Prabhakaran, P. \& Bajohr, S. 2016 Review on methanation - From fundamentals to current projects. Fuel 166, 276-296.

Sanchez-Palencia, E. 1980 Non-Homogeneous Media and Vibration Theory, Lecture Notes in Physics, vol. 127. Springer Berlin Heidelberg.

Shepel, S. V., Wakili, K. Ghazi \& Hugi, E. 2010 Vapor convection in gypsum plasterboard exposed to fire: Numerical simulation and validation. Numerical Heat Transfer, Part A: Applications 57, 911-935.

Shreve, R. L. 1967 Migration of air bubbles, vapor figures, and brine pockets in ice under a temperature gradient. Journal of Geophysical Research 72, 4093-4100.

Slattery, J. C. 1967 General balance equation for a phase interface. Industrial \& Engineering Chemistry Fundamentals 6 (1), 108-115.

Spagnolie, S. E. \& Lauga, E. 2012 Hydrodynamics of self-propulsion near a boundary: predictions and accuracy of far-field approximations. Journal of Fluid Mechanics 700, $105-147$.

Vetter, R., Sigg, S., Singer, H. M., Kadau, D., Herrmann, H. J. \& Schneebeli, M. 2010 Simulating isothermal aging of snow. EPL (Europhysics Letters) 89, 26001.

Vu, H. T. \& Tsotsas, E. 2018 Mass and heat transport models for analysis of the drying process in porous media: A review and numerical implementation. International Journal of Chemical Engineering 2018, 9456418,

Warning, A. D., Arquiza, J.M.R. \& Datta, A. K. 2015 A multiphase porous medium transport model with distributed sublimation front to simulate vacuum freeze drying. Food and Bioproducts Processing 94, 637-648.

Whitaker, S. 1986 Flow in porous media I: A theoretical derivation of Darcy's law. Transport in Porous Media 1 (1), 3-25.

Whitaker, S. 1999 The Method of Volume Averaging. Springer Netherlands. 